\title{
WILD-seq: Clonal deconvolution of transcriptomic signatures of sensitivity and resistance to cancer therapeutics in vivo.
}

\author{
Sophia A Wild ${ }^{1} \dagger$, Ian G Cannell ${ }^{1} \dagger$, Katarzyna Kania ${ }^{1}$, CRUK IMAXT Grand Challenge Team ${ }^{1}$, Gregory \\ J Hannon $^{1 *}$, Kirsty Sawicka ${ }^{1 *}$ \\ ${ }^{1}$ Cancer Research UK Cambridge Institute, University of Cambridge, Li Ka Shing Centre, Robinson \\ Way, Cambridge. CB2 ORE. UK. \\ $\dagger$ these authors contributed equally to this work
}

* to whom correspondence should be addressed Greg.Hannon@cruk.cam.ac.uk or Kirsty.Sawicka@cruk.cam.ac.uk

\begin{abstract}
Tumour heterogeneity is thought to be a major barrier to successful cancer treatment due to the presence of drug resistant clonal lineages. However, identifying the characteristics of such lineages that underpin resistance to therapy has remained challenging. Here we present WILD-seq; Wholistic Interrogation of Lineage Dynamics by sequencing, a platform that leverages expressed barcodes to simultaneously map clonal identities and transcriptional states at single cell resolution. Our optimised pipeline ensures recurrent representation of clonal lineages across animals and samples, facilitating analysis of clonal dynamics under perturbation. Application of WILD-seq to two triple negative mammary carcinoma mouse models, identified changes in clonal abundance, gene expression and microenvironment in response to JQ1 or taxane chemotherapy. WILD-seq reveals oxidative stress protection as a major mechanism of taxane resistance that renders our tumour models collaterally sensitive to non-essential amino acid deprivation. In summary, WILD-seq enables facile coupling of lineage and gene expression in vivo to elucidate clone-specific pathways of resistance to cancer therapies.
\end{abstract}

\section{Introduction}

Evolution of clonal architecture is a key feature of cancer progression, enabling tumours to adapt to selective pressures imposed by therapeutic intervention and providing fuel for therapy resistance ${ }^{1,2}$. Resistance can arise through the selection of rare pre-existing clones or through the adaptation of specific clonal lineages that may display enhanced plasticity. Being able to identify mechanisms of resistance in vivo in heterogenous, polyclonal cancer cell populations is therefore imperative for the development of rational combination therapy regimens.

A key challenge to understanding the complex response of heterogeneous populations to therapeutic interventions, is being able to identify and follow individual clonal lineages within a tumour over the course of treatment and perform, in parallel, molecular characterisation of these populations. Importantly, it is critical to assess the unperturbed state to understand what pre-existing characteristics may have conferred the selected phenotype. Retrospective lineage tracing approaches can infer phylogenetic relationships of cells within a tumour by using naturally heritable marks, such as singlenucleotide polymorphisms (SNVs) or copy-number variations $(\mathrm{CNVs})^{3}$. These approaches are particularly powerful for studying the clonal dynamics in patient samples and have yielded tremendous insights into the importance of genomic heterogeneity to tumour progression and resistance to therapy ${ }^{4-}$ 7. However, since lineage identity cannot be readily coupled to molecular phenotypes such a gene expression, such methods have limited applicability to the identification of causative mechanisms of resistance that could be exploited to improve patient outcomes.

Recent technological advances that utilise heritable genetic barcodes with single-cell RNA sequencing provide powerful tools to integrate complementary information about cell lineage and gene expression at the single cell level ${ }^{8-12}$. To date, application of these techniques to the study of tumour heterogeneity and therapeutic resistance mechanisms has largely been confined to the study of tumour cells in culture. Expansion of such integrative methods to mouse models of cancer has the potential to provide a tractable system for the study of tumour heterogeneity in the context of physiological levels of oxygen, growth factors, metabolites, and cells of the microenvironment all of which can have a major impact on therapeutic outcome. 
Here we present, WILD-seq (Wholistic Interrogation of Lineage Dynamics by sequencing), an accessible and adaptable platform for lineage tracing at the single-cell transcriptomic level that facilitates in vivo analysis of clonal dynamics. Our optimised pipeline ensures recurrent representation of clonal lineages across animals and samples, facilitating analysis of clonal dynamics under the selective pressure of therapeutic intervention. We demonstrate the utility of WILD-seq to simultaneously identify changes in clonal abundance, gene expression and the composition of the tumour microenvironment in response to transcriptional perturbation with the BET bromodomain inhibitor, JQ1. Importantly, when applied to the identification of determinants of sensitivity and resistance to taxane chemotherapy across two triple negative mammary carcinoma mouse models, WILD-seq revealed signatures of resistance present in clonal lineages prior to chemotherapy and enriched in our models and patient samples after chemotherapy. We further demonstrate the potential for such discoveries to elucidate new combination therapies.

\section{Results}

\section{Establishment of an expressed barcode system to simultaneously detect clonal lineage and gene expression.}

WILD-seq uses a lentiviral library to label cells with an expressed, heritable barcode that enables identification of clonal lineage in conjunction with single cell RNA sequencing. The WILD-seq construct comprises a zsGreen transcript which harbours in its 3' untranslated region (UTR) a barcode consisting of two 12 nucleotide variable regions separated by a constant linker (Fig. 1a). Each variable region is separated from any other sequence in the library by a Hamming distance of 5 to allow for library preparation and sequencing error correction and our library contains over 1.5 million unique barcodes. The barcode is appropriately positioned relative to the polyadenylation signal to ensure its capture and sequencing by standard oligo-dT single cell sequencing platforms.

The standard WILD-seq pipeline is illustrated in Figure 1b. A heterogeneous cell line is transduced with a barcode library at low multiplicity of infection (MOI) to ensure that each cell receives a maximum of one barcode. An appropriate size pool of barcoded clones is selected and stabilized in culture. Empirically, we have found a pool established from 750 individual clones works well to provide effective representation of the diversity within the cell line while also enabling recurrent representation of the same clones across animals and experiments. Once stabilized in culture, the pool of WILD-seq clones can be analysed directly by single cell sequencing or injected into a recipient animal for in vivo tumour growth. WILD-seq single cell sequencing libraries can be prepared using a standard oligo-dT based protocol and addition of an extra PCR amplification step can be used to increase coverage of the barcode region and aid cell lineage assignment.

We first established a WILD-seq clonal pool from the mouse 4T1 cell line, a triple negative mammary carcinoma model that can be orthotopically implanted into the mammary fat pad of a BALB/c syngeneic host, which we have previously shown to be heterogeneous with distinct sub-clones having unique biological properties ${ }^{13}$. We performed single cell sequencing of the in vitro WILD-seq pool (Fig. 1c) and in vivo tumours derived from this clonal pool (Fig. 1d). Over the course of our studies, we injected multiple cohorts of mice with our WILD-seq 4T1 pool as detailed in Supplementary Table 1, some of which were subjected to a specific drug regime. All tumours were harvested at humane endpoint as determined by tumour volume and immediately dissociated for single cell sequencing.

For the purpose of characterising the baseline properties of our clones, we performed an in-depth transcriptomic analysis of all tumours from untreated and vehicle-treated animals. A WILD-seq barcode and thereby clonal lineage could be unambiguously assigned to $30-60 \%$ of cells per sample within the presumptive tumour cell/mammary epithelial cell cluster. 132 different WILD-seq barcodes were observed in vitro and in total 94 different WILD-seq barcodes were observed across our in vivo tumour samples. Our in vivo tumour samples comprised both tumour cells and host cells of the tumour microenvironment including cells of the innate and adaptive immune system, enabling simultaneous profiling of the tumour and its microenvironment (Fig. 1d). Clustering was performed after removal of reads mapping to the WILD-seq vector, to avoid any influence of the WILD-seq transcript on clustering, and the WILD-seq barcode assignment subsequently overlaid onto these data. The tumour cell clusters were clearly identifiable by the high expression of the barcode transcript. Occasionally a barcode was observed in cells which clustered according to their transcriptome outside of the main tumour cluster. Since this could be the result of sequencing or technical error causing a mismatch between the WILD- 
seq barcode and the cell of origin, only barcoded cells that clustered within the main tumour/mammary epithelium cell cluster were included in our analysis.

We reproducibly observed the same clonal populations across animals and independent experiments which is critical to our ability to examine the effects of different interventions and treatments (Fig. 1d, 1e). The relative abundance of clones was similar in tumours grown in NOD scid gamma (NSG) immunodeficient and BALB/c immunocompetent mice but was drastically different to that found in the in vitro cell pool from which they were established (Fig. 1e, Supplementary Table 2), suggesting that clones that show greatest fitness in cell culture do not necessarily show fitness in vivo. Therefore, in vitro clonal lineage tracking experiments are likely to capture a different collection of clones and have the potential to identify sensitive or resistance clones that are not represented in vivo. Pseudo-bulk analysis of the major clonal lineages revealed that the composition of the tumour microenvironment has a dramatic effect on the transcriptome of the tumour cells for all clones (Fig. 1f). Comparison of in vitro culture, tumours from NSG mice, and tumours from BALB/c mice by principal component analysis (PCA), showed clear separation of the tumour cells depending on their environment, with differences in interferon gamma signaling, TNF-alpha signaling, and cell cycle being most prominent between cells grown in vivo and in vitro (PC1, Fig. 1g). Differences in gene expression between tumours growing in immunocompetent and immunodeficient hosts were related to changes in the expression of extracellular matrix proteins and changes in interferon gamma and II-2 signaling, consistent with the differences in T-cell abundance (PC2, Fig. 1g). These data highlight the importance of the host immune system in sculpting the transcriptome and provide cautionary context for the analysis of tumour gene expression in immune-compromised hosts. Although there were large differences between clonal gene expression patterns across hosts the clones showed consistent differences in gene expression across all settings, reflective of intrinsic clonal properties, with the biggest variation in gene expression across the clones being related to their position along the epithelial-mesenchymal transition (EMT) axis (PC3, Fig. 1g). In particular, Clone 679 is the most distinct and the most mesenchymal of the clones.

To further characterise the major clones in our tumours, we performed gene set expression analysis using AUCell ${ }^{14}$ to identify pathways that are enriched in cells of a specific clonal lineage. Analysis was performed across four independent experiments each with three vehicle-treated animals and for the majority of clones we were able to identify distinct gene expression signatures that were reproducible across animals and experiments (Fig. 1h, Supplementary Table 4, Supplementary Table 5).

\section{Simultaneous detection of changes in clonal abundance, gene expression, and tumour microenvironment in response to BET bromodomain inhibition with WILD-seq.}

Having established that we can repeatedly observe the same clonal lineages and their gene expression programs across animals and experiments, we next sought to perturb the system. We chose the BET bromodomain inhibitor JQ1 for our proof-of-principle experiments to assess the ability of the WILD-seq system to simultaneously measure changes in clonal abundance, gene expression and the tumour microenvironment that occur following therapeutic intervention. JQ1 competitively binds to acetylated lysines, displacing BRD4 and thereby repressing transcription at specific loci. A large number of studies have indicated that BET inhibitors may be beneficial in the treatment of hematopoietic malignancies and solid tumours including breast cancer, possibly by inhibiting certain key proto-oncogenes such as MYC ${ }^{15}$.

Treatment of our 4T1 WILD-seq tumour-bearing mice with JQ1 caused an initial suppression of tumour growth but with only a small overall effect on time to humane endpoint (Fig. 2a). Tumours treated with JQ1 or vehicle alone were harvested at endpoint, dissociated and subjected to single cell sequencing (Fig. 2b). Two independent experiments were performed, each with 3 mice per condition.

We first explored whether JQ1 had any effect on the tumour microenvironment. The most striking difference we observed was a change in abundance among the cells belonging to the T-cell compartment. To analyse this further, we computationally extracted these cells from the single cell data, reclustered them and performed differential abundance testing using Milo (Fig. 2c). Milo detects sets of cells that are differentially abundant between conditions by modeling counts of cells in neighborhoods of a KNN graph ${ }^{16}$. When applied to our reclustered T-cells, Milo identified a significant decrease in abundance in cytotoxic T-cells, as identified by their expression of Cd8a and Cd8b1, following JQ1 treatment. A significant change was observed in both of our experiments although the magnitude of the effect was greater in experiment $A$.

We next examined the effect of JQ1 treatment on the transcriptome of the tumour cells. Differential expression analysis was performed for each clonal lineage and experiment independently. As expected, given its mode of action, we identified significant down-regulation of a wide range of genes with 
consistent changes across clonal lineages (Fig 2d, supplementary table 6). Among the repressed genes, were a number of genes related to interferon (IFN) signaling and antigen processing and presentation (Fig. 2d, 2e), including GBP2 which is strongly induced by IFN gamma, the MHC class II protein, Cd74, and B2m, a component of the MHC class I complex. JQ1 has previously been reported to directly inhibit transcription of IFN-response genes ${ }^{17,18}$ suggesting this may be due to the direct action of JQ1 within our tumour cells, however JQ1-dependent changes to the tumour microenvironment may also influence these expression pathways.

Our barcoded $4 \mathrm{~T} 1$ clones showed varied sensitivity to JQ1, with treatment causing reproducible changes to clonal proportions within the tumour (Fig. 2f, 2g, Supplementary Table 2). In particular, one of the most abundant clones, clone 473 , is highly sensitive to JQ1 treatment. In contrast, 3 clones were identified as being the most resistant to JQ1 treatment, clones 93, 439 and 264. These clones which together make up less than $5 \%$ of the tumour in vehicle treated mice constitute on average $12.8 \%$ of the JQ1-treated tumours. To examine baseline transcriptomic signatures of JQ1-sensitivity and resistance, we identified gene sets whose expression in vehicle-treated tumours was highly correlated with response (Figs. 2h, 2i, Supplementary Table 7). Interestingly, interferon signaling which is significantly attenuated in our JQ1-treated tumours is highly correlated with sensitivity to JQ1, suggesting a possible higher dependence of the sensitive clones on these pathways. Conversely resistance is associated with higher levels of unfolded protein response and mTOR signaling consistent with a known role of mTOR-mediated autophagy in resistance to JQ1 ${ }^{19}$, and cytotoxic synergy between $\mathrm{PI} 3 \mathrm{~K} / \mathrm{mTOR}$ inhibitors and BET inhibitors ${ }^{20,21}$.

\section{Clonal transcriptomic correlates of response and resistance to taxane chemotherapy in the 4T1 mammary carcinoma model.}

Our studies with JQ1 exemplify the ability of the WILD-seq system to simultaneous measure in vivo the effect of therapeutic intervention on clonal dynamics, gene expression and tumour microenvironment. However, we were interested in using our system to investigate a chemotherapeutic agent currently in use in the clinic. We therefore treated our 4T1 WILD-seq tumour-bearing mice with docetaxel as a representative taxane, a class of drugs which are routinely used to treat triple negative breast cancer patients. As with JQ1, docetaxel treatment resulted in an initial, modest reduction in tumour growth followed by relapse (Fig. 3a). Comparison of vehicle and docetaxel (DTX) treated tumors revealed differential response of clonal lineages to treatment (Figs. 3b, 3c, 3d, Supplementary Table 2) with clone 679 being the most resistant and clone 238 the most sensitive to chemotherapy.

Correlating the clones' baseline transcriptomic profiles with response to docetaxel, revealed a major role for EMT in modulating sensitivity and resistance to taxane-based therapy. The 4T1 clones which are most sensitive to docetaxel are characterised by high expression of E-Cadherin regulated genes and low Zeb1 activity consistent with a more epithelial phenotype (Figs. 3e, 3f, Supplementary Table 8). These observations are in agreement with previous studies that have implicated EMT, and its associated endowment of cancer stem cell-like characteristics, as a mechanism of resistance to cytotoxic chemotherapies like docetaxel in cell culture and patients ${ }^{22-24}$. Resistance to docetaxel was correlated with up-regulation of multiple gene sets (Figs. 3e, 3f, Supplementary Table 8). This included genes whose expression is elevated in non-responders to docetaxel in human breast cancer patients ${ }^{25}$ demonstrating the relevance of our findings. Interestingly, we also identify metabolic reprogramming as a potential mechanism of docetaxel resistance with higher expression of genes related to glycogen and glutathione metabolism being correlated with resistance to docetaxel (Fig, 3e).

\section{Clonal transcriptomic signatures of response and resistance to taxane chemotherapy in the D2A1 mammary carcinoma model.}

To explore the general applicability of WILD-seq to other models, we utilised a second triple negative mammary carcinoma model, D2A1-m2 (hereafter referred to as D2A1). Similar to the 4T1 cell line, this line was derived from a mouse mammary tumour in a BALB/c mouse and can be orthotopically implanted into the mammary fat pad of immunocompetent, syngeneic hosts ${ }^{26}$.

We established a WILD-seq D2A1 clonal pool by transducing the D2A1 cell line with our WILD-seq barcode library. These barcoded cells were orthotopically implanted into a cohort of mice, half of which were treated with docetaxel, while the remaining animals received vehicle alone. Docetaxel treatment caused an initial reduction in tumour growth with subsequent relapse (Fig. 4a). We performed single cell RNA sequencing of three tumours per condition and assigned the tumour cells to a distinct clonal lineage based on the presence of the WILD-seq barcode (Fig. 4b). In total 103 different WILD-seq barcodes were observed in vivo with a dramatic shift in relative clonal abundance on docetaxel treatment (Fig. 4d, Supplementary Table 3). Unlike our 4T1 breast cancer model, variation between 
clonal lineages was no longer dominated by the EMT status of the clones and all clones exhibited a more mesenchymal-like phenotype consistent with the fact that this was a subline of D2A1 selected for its metastatic properties (Fig. 4c). This provides us with a distinct yet complementary system to investigate chemotherapy resistance with the potential to reveal alternative mechanisms than EMT status.

We identified 3 clones which were acutely sensitive to docetaxel, clones 118, 2874 and 1072. Together these constitute on average $37 \%$ of the vehicle-treated tumours but only $1.3 \%$ of the docetaxel-treated tumours (Fig. 4d). To understand the properties of these clones, we analysed the baseline gene expression characteristics of clones in vehicle-treated tumours. The gene expression of cells from a clone of interest was compared to all tumour cells to which a WILD-seq barcode could be assigned from the same sample, and clonal signatures identified that were significantly enriched across animals. Specific gene expression signatures were identifiable for all clones analysed, some of which were unique to a single clone while others overlapped across the sensitive clones (Fig. 4e, Supplementary Table 9, Supplementary Table 10). For example, clone 1072 shows elevated levels of expression of cell cycle related pathways, such as E2F-target genes (Fig 4f), indicating that aberrant cell cycle control in these cells that could increase their susceptibility to an antimitotic cancer drug (Fig. 4f), interestingly high levels of E2F-targets have recently been shown to be associated with response to chemotherapy in breast cancer ${ }^{27}$.

Three clones robustly increased their relative abundance within the tumour following docetaxel treatment, clones 1197, 751 and 1240, which despite making up less than $1 \%$ of the vehicle-treated tumours together constituted more than $20 \%$ of the docetaxel-treated tumours (Fig. $4 \mathrm{~d}$ ). Due to the low abundance of cells in vehicle-treated samples, cells belonging to all 3 clones were pooled to analyse their baseline gene expression profiles (Fig. 4g). Among the gene sets differentially expressed between resistant and sensitive clones, were a number of breast cancer amplicons indicating that there may be specific copy number variations associated with these clones (Figs. 4g, 4h). However single cell DNA sequencing data would be required to confirm the presence of specific genetic traits within our clones. Interestingly, gains in $8 q 24^{28}, 20 q 11^{29}$ and loss of $16 q^{30}$ have previously been report to be associated with resistance to taxane-based chemotherapy in agreement with our findings. Highly upregulated within all 3 of our resistant clones were genes related to the NRF2 pathway, even in the absence of docetaxel treatment (Figs. 4g, 4h). NRF2 activation has been linked to cancer progression and metastasis and has been suggested to confer resistance to chemotherapy ${ }^{31-36}$.

\section{Delineating the contribution of clonal abundance to gene expression changes upon drug treatment.}

Prior to the advent of single cell sequencing, the majority of studies relied on bulk RNA-seq or microarray analysis of gene expression to interrogate the effect of chemotherapeutic interventions. While informative, these studies cannot differentiate between changes in bulk gene expression that arise due to clonal selection and changes that are induced within a clonal lineage as the result of drug exposure. Even with single cell sequencing, definitive identification of the same clonal population across treatment conditions is impractical. Our method alleviates these difficulties by enabling the direct comparison of clones of the same lineage under different conditions.

To examine the relative contribution of clonal selection and transcriptional reprogramming to changes in gene expression upon chemotherapy, we compared analysis of gene expression within each clone individually to a combined analysis of all pooled tumour cells (Fig. 5, Supplementary Table 11). Consistent with their mode of action, docetaxel had relatively little effect on the transcriptome of individual clones while JQ1 caused substantial changes to the transcriptome predominantly downregulating gene expression. Genes were identified under all treatments that were altered within the tumour as a whole but as a result of clonal selection rather than intra-clonal changes in gene expression, with the biggest effects being observed with docetaxel treatment in D2A1 tumours, in agreement with this condition inducing the largest changes in relative clonal abundance. To confirm that changes in gene expression detected in bulk tumour analysis but not the clonal analysis could be attributed to differences in clonal sensitivity to chemotherapy, we analysed baseline expression of these genes across the major clonal populations (Fig. 5b). As expected, we found that genes up-regulated only in bulk tumour analysis had significantly higher expression in clones resistant to chemotherapy (that increase in abundance with treatment) and genes only down-regulated in bulk tumour analysis had significantly lower expression in these resistant clonal lineages.

Among the genes that change in expression within the tumour as a whole as a result of clonal selection upon docetaxel treatment, we identified a number of genes related to glutathione synthesis and 
conjugation including Mgst2, Esd and Gclm, that may endow resistant clones with greater ability to resolve reactive oxygen species (ROS) induced by docetaxel ${ }^{37}$. Of note, we also observed that in 4T1 tumours, Epcam was significantly reduced in expression in the bulk tumour but was not changed within the individual clonal populations. This suggests that rather than inducing an EMT within the tumour cells, docetaxel is selecting clones of a pre-existing more mesenchymal phenotype.

\section{Convergent WILD-seq analysis across models identifies redox defense as a mediator of taxane resistance.}

To examine if there were any shared mechanisms of taxane resistance across our 4T1 and D2A1 WILDseq clones, we looked for genes that were enriched in resistant clonal lineages in both models. We identified 47 overlapping resistance genes (Fig. 6a, Supplementary Table 12). These genes were significantly enriched in pathways related to resolution of oxidative stress including the NRF2 pathway and glutathione-mediated detoxification (Fig. 6b).

Importantly, these genes were also enriched in human patients following combined anthracycline and taxane-based therapy, highlighting the potential clinical significance of our findings (Fig. 6c). Gene expression data from a previously published study with paired pre-neo adjuvant chemotherapy (NAC) core needle biopsies and post-chemotherapy surgical samples ${ }^{38}$ was re-analysed using GSVA $^{39}$ to determine the effect of chemotherapy on a gene set composed of our 47 shared resistance genes (Fig. $6 \mathrm{c}$ ) as well as NRF2-targets as determined by ChIP enrichment analysis (CHEA) ${ }^{40}$ (Fig. 6d). Expression of both these gene sets was significantly increased after chemotherapy, which our data would suggest is the result of outgrowth of resistant clonal lineages with increased propensity to withstand taxaneinduced oxidative stress.

Given these findings, we hypothesised that combining taxane-based chemotherapy with a drug specifically targeting resistant clones with high NRF2 signaling would provide a highly effective treatment regime. To test this hypothesis, we leveraged the finding that tumours with constitutively active Nrf2, due to mutation in the negative regulator Keap1, have metabolic vulnerabilities that arise from their high antioxidant production ${ }^{36}$, including dependency of glutamine ${ }^{36}$ and a general dependency on exogenous non-essential amino acids (NEAA) including asparagine ${ }^{41}$. This metabolic dependency can be targeted therapeutically by L-asparaginase (from E.coli), an enzyme that is used in the clinical management of acute lymphoblastic leukemia (ALL) ${ }^{42}$, that catalyzes the conversion of Lasparagine to aspartic acid and ammonia but also has glutaminase activity that may contribute to its therapeutic effects in ALL ${ }^{43}$. To ascertain whether docetaxel resistant clones were collaterally sensitive to L-asparaginase we treated D2A1 WILD-seq tumours with docetaxel alone for one week, to select for resistant clones, then in combination with L-asparaginase for half of the second week, followed by maintenance dosing of L-asparaginase alone. As shown in Figure $6 \mathrm{e}$, the addition of L-asparaginase caused a precipitous drop in tumour volume, relative to docetaxel treatment alone, that was maintained until the vehicle treated animals reached humane endpoint (Fig. 6e). Importantly, L-asparaginase alone had no significant effect on tumour growth, indicative of a docetaxel-induced effect. Further drug schedule optimization is required since the combination at the doses used here had increased toxicity compared to either drug alone. However, these data support the notion that WILD-seq can identify causal mechanisms of drug resistance in vivo, that be leveraged to inform new combination therapies. Since these redox defense signatures are detectable in patients after neo-adjuvant chemotherapy (NAC), one can envisage an approach whereby patients receiving NAC have the surgical tumour specimen profiled for NRF2 gene signatures and those with high levels receive a post-operative course of L-asparginase.

\section{Discussion}

Tumour heterogeneity is thought to underlie drug resistance through the selection of clonal lineages that can preferentially survive therapy. However, identifying the features of such lineages, so that they can be targeted therapeutically, has been challenging due the lack of understanding of their molecular characteristics and the lack of animal models to prospectively test therapeutic interventions and combinations thereof. To overcome these challenges, we have developed WILD-seq, a system that leverages expressed barcodes, population bottle necking, syngeneic mouse models and single cell RNA-seq to link clonal lineage to the transcriptome. Although a number of variations of expressed barcode systems exist ${ }^{8-12}$, our approach is unique in that we purposefully bottleneck our clonal population to achieve a balance between maximizing clonal diversity and minimizing variation in clonal representation across replicate animals and experiments. It is this feature that allows us to robustly call 
clonal gene expression signatures and differential clonal abundance before and after therapeutic intervention and it is this in turn that allows us to identify relevant drug resistance mechanisms in vivo.

We find that the abundance of clones in cell culture and in vivo differ greatly, with the most abundant clones in vitro being lowly represented in vivo and vice versa thus providing a cautionary note when analyzing drug response in vitro. We deployed WILD-seq to determine changes in clonal architecture and the microenvironment in response to transcriptional perturbation with JQ1 and analysed sensitivity and resistance to taxane chemotherapy in two syngeneic, triple negative, mammary carcinoma models highlighting both known and new pathways of resistance ${ }^{44}$. Resistance to cancer therapies can arise due to clonal selection or through adaptive reprograming of the epigenome and transcriptome of individual clones. Our data with docetaxel treatment in 4T1 and D2A1 indicate that over the time frames we have examined clonal selection is the dominant force driving resistance to chemotherapy with gene expression signatures, such as EMT and Nrf2 signaling, being present in clones at baseline that are then selected for during therapy. However, recurrent representation of clonal lineages across treatments should enable identification of transcriptional reprogramming should it exist with other therapeutic modalities or model systems using the WILD-seq platform.

Applying WILD-seq to examine docetaxel response across two TNBC models afforded the opportunity to overlap resistance genes for the same drug across models and remove model-specific effects. These analyses uncovered a critical role for redox defense in docetaxel resistance that also appears to be operative in human breast cancer patients after chemotherapy. In light of previous work linking constitutive Nrf2 signaling to a dependency on exogenous non-essential amino acids (NEAAs) ${ }^{41}$ we exploited this property of docetaxel resistant clones by treating tumours first with docetaxel then with Lasparaginase revealing a profound suppression of tumour growth compared to docetaxel alone. Interestingly, we have previously shown that asparagine bioavailability regulates EMT and metastatic progression in breast cancer ${ }^{45}$. Thus, asparagine deprivation may present multiple benefits to breast cancer patients. With respect to chemotherapy response, the data presented here suggest that docetaxel resistant clones exhibit a phenomenon called collateral sensitivity ${ }^{46}$, first described for antibiotics $^{47}$, where resistance to one drug comes at the cost of sensitivity to a second drug. In the context of cancer and taxanes, clonal collateral sensitivity has the distinct advantage over other therapeutic strategies of maintaining the initial first line therapy and only modifying subsequent therapies. Taken together our data demonstrate that WILD-seq is a robust platform for identifying clonal determinants of response to therapy in vivo that can be leveraged to inform new combination therapies.

\section{References}

1. Turajlic, S. \& Swanton, C. Metastasis as an evolutionary process. Science 352, 169-175 (2016).

2. Bhang, H. C. et al. Studying clonal dynamics in response to cancer therapy using high-complexity barcoding. Nat Med 21, 440-448 (2015).

3. Schwartz, R. \& Schäffer, A. A. The evolution of tumour phylogenetics: principles and practice. Nat Rev Genet 18, 213-229 (2017).

4. Jamal-Hanjani, M. et al. Tracking Genomic Cancer Evolution for Precision Medicine: The Lung TRACERx Study. Plos Biol 12, e1001906 (2014).

5. Gerlinger, M. et al. Intratumor Heterogeneity and Branched Evolution Revealed by Multiregion Sequencing. New Engl J Medicine 366, 883-892 (2012).

6. Landau, D. A. et al. Evolution and Impact of Subclonal Mutations in Chronic Lymphocytic Leukemia. Cell 152, 714-726 (2013).

7. Ding, L. et al. Clonal evolution in relapsed acute myeloid leukaemia revealed by whole-genome sequencing. Nature 481, 506-510 (2012). 
8. Quinn, J. J. et al. Single-cell lineages reveal the rates, routes, and drivers of metastasis in cancer xenografts. Science 371, eabc1944 (2021).

9. Biddy, B. A. et al. Single-cell mapping of lineage and identity in direct reprogramming. Nature 564, 219-224 (2018).

10. Simeonov, K. P. et al. Single-cell lineage tracing of metastatic cancer reveals selection of hybrid EMT states. Cancer Cell 39, 1150-1162.e9 (2021).

11. Weinreb, C., Rodriguez-Fraticelli, A., Camargo, F. D. \& Klein, A. M. Lineage tracing on transcriptional landscapes links state to fate during differentiation. Science 367, eaaw3381 (2020).

12. Gutierrez, C. et al. Multifunctional barcoding with ClonMapper enables high-resolution study of clonal dynamics during tumor evolution and treatment. Nat Cancer 2, 758-772 (2021).

13. Wagenblast, E. et al. A model of breast cancer heterogeneity reveals vascular mimicry as a driver of metastasis. Nature 520, 358-62 (2015).

14. Aibar, S. et al. SCENIC: single-cell regulatory network inference and clustering. Nat Methods 14, 1083-1086 (2017).

15. Jiang, G., Deng, W., Liu, Y. \& Wang, C. General mechanism of JQ1 in inhibiting various types of cancer. Mol Med Rep 21, 1021-1034 (2020).

16. Dann, E., Henderson, N. C., Teichmann, S. A., Morgan, M. D. \& Marioni, J. C. Differential abundance testing on single-cell data using k-nearest neighbor graphs. Nat Biotechnol 1-9 (2021) doi:10.1038/s41587-021-01033-z.

17. Gibbons, H. R. et al. Bromodomain inhibitor JQ1 reversibly blocks IFN-y production. Sci Rep-uk 9, 10280 (2019).

18. Gusyatiner, O. et al. BET inhibitors repress expression of Interferon-stimulated genes and synergize with HDAC inhibitors in glioblastoma. Neuro-oncology 23, noab115- (2021).

19. Luan, W. et al. Akt/mTOR-Mediated Autophagy Confers Resistance To BET Inhibitor JQ1 In Ovarian Cancer. Oncotargets Ther 12, 8063-8074 (2019).

20. Stratikopoulos, E. E. et al. Kinase and BET Inhibitors Together Clamp Inhibition of PI3K Signaling and Overcome Resistance to Therapy. Cancer Cell 27, 837-851 (2015).

21. Lee, D. H. et al. Synergistic effect of JQ1 and rapamycin for treatment of human osteosarcoma. Int J Cancer 136, 2055-2064 (2015).

22. Creighton, C. J. et al. Residual breast cancers after conventional therapy display mesenchymal as well as tumor-initiating features. Proc National Acad Sci 106, 13820-13825 (2009).

23. Gupta, P. B. et al. Identification of Selective Inhibitors of Cancer Stem Cells by High-Throughput Screening. Cell 138, 645-659 (2009).

24. Bhola, N. E. et al. TGF- $\beta$ inhibition enhances chemotherapy action against triple-negative breast cancer. J Clin Invest 123, 1348-1358 (2013).

25. Honma, K. et al. RPN2 gene confers docetaxel resistance in breast cancer. Nat Med 14, 939-948 (2008). 
26. Jungwirth, U. et al. Generation and characterisation of two D2A1 mammary cancer sublines to model spontaneous and experimental metastasis in a syngeneic BALB/c host. Dis Model Mech 11, dmm031740 (2017).

27. Sammut, S.-J. et al. Multi-omic machine learning predictor of breast cancer therapy response. Nature 1-10 (2021) doi:10.1038/s41586-021-04278-5.

28. Han, S. et al. Genomic change of chromosome 8 predicts the response to taxane-based neoadjuvant chemotherapy in node-positive breast cancer. Oncol Rep 24, 121-8 (2010).

29. VOUTSADAKIS, I. A. Chromosome 20q11.21 Amplifications in Colorectal Cancer. Cancer Genom Proteom 18, 487-496 (2021).

30. Höglander, E. K. et al. Time series analysis of neoadjuvant chemotherapy and bevacizumabtreated breast carcinomas reveals a systemic shift in genomic aberrations. Genome Med 10, 92 (2018).

31. Konstantinopoulos, P. A. et al. Keap1 Mutations and Nrf2 Pathway Activation in Epithelial Ovarian Cancer. Cancer Res 71, 5081-5089 (2011).

32. Shibata, T. et al. Genetic Alteration of Keap1 Confers Constitutive Nrf2 Activation and Resistance to Chemotherapy in Gallbladder Cancer. Gastroenterology 135, 1358-1368.e4 (2008).

33. Homma, S. et al. Nrf2 Enhances Cell Proliferation and Resistance to Anticancer Drugs in Human Lung Cancer. Clin Cancer Res 15, 3423-3432 (2009).

34. Jiang, T. et al. High Levels of Nrf2 Determine Chemoresistance in Type II Endometrial Cancer. Cancer Res 70, 5486-5496 (2010).

35. Singh, A. et al. Dysfunctional KEAP1-NRF2 Interaction in Non-Small-Cell Lung Cancer. Plos Med 3 , e420 (2006).

36. Romero, R. et al. Keap1 loss promotes Kras-driven lung cancer and results in dependence on glutaminolysis. Nat Med 23, 1362-1368 (2017).

37. Alexandre, J., Hu, Y., Lu, W., Pelicano, H. \& Huang, P. Novel Action of Paclitaxel against Cancer Cells: Bystander Effect Mediated by Reactive Oxygen Species. Cancer Res 67, 3512-3517 (2007).

38. Vera-Ramirez, L. et al. Transcriptional Shift Identifies a Set of Genes Driving Breast Cancer Chemoresistance. Plos One 8, e53983 (2013).

39. Hänzelmann, S., Castelo, R. \& Guinney, J. GSVA: gene set variation analysis for microarray and RNA-Seq data. Bmc Bioinformatics 14, 7-7 (2013).

40. Lachmann, A. et al. ChEA: transcription factor regulation inferred from integrating genome-wide ChIP-X experiments. Bioinformatics 26, 2438-2444 (2010).

41. LeBoeuf, S. E. et al. Activation of Oxidative Stress Response in Cancer Generates a Druggable Dependency on Exogenous Non-essential Amino Acids. Cell Metab 31, 339-350.e4 (2020).

42. Batool, T., Makky, E. A., Jalal, M. \& Yusoff, M. M. A Comprehensive Review on I-Asparaginase and Its Applications. Appl Biochem Biotech 178, 900-923 (2016).

43. Chan, W.-K. et al. Glutaminase Activity of L-Asparaginase Contributes to Durable Preclinical Activity against Acute Lymphoblastic Leukemia. Mol Cancer Ther 18, 1587-1592 (2019). 
bioRxiv preprint doi: https://doi.org/10.1101/2021.12.09.471927; this version posted December 10, 2021. The copyright holder for this preprint (which was not certified by peer review) is the author/funder, who has granted bioRxiv a license to display the preprint in perpetuity. It is made available under aCC-BY 4.0 International license.

44. Marine, J.-C., Dawson, S.-J. \& Dawson, M. A. Non-genetic mechanisms of therapeutic resistance in cancer. Nat Rev Cancer 20, 743-756 (2020).

45. Knott, S. R. V. et al. Asparagine bioavailability governs metastasis in a model of breast cancer. Nature 554, 378-381 (2018).

46. Roemhild, R. \& Andersson, D. I. Mechanisms and therapeutic potential of collateral sensitivity to antibiotics. Plos Pathog 17, e1009172 (2021).

47. Imamovic, L. \& Sommer, M. O. A. Use of Collateral Sensitivity Networks to Design Drug Cycling Protocols That Avoid Resistance Development. Sci Transl Med 5, 204ra132-204ra132 (2013).

48. Stuart, T. et al. Comprehensive Integration of Single-Cell Data. Cell 177, 1888-1902.e21 (2019).

49. Subramanian, A. et al. Gene set enrichment analysis: A knowledge-based approach for interpreting genome-wide expression profiles. Proc National Acad Sci 102, 15545-15550 (2005).

50. Liberzon, A. et al. Molecular signatures database (MSigDB) 3.0. Bioinformatics $27,1739-1740$ (2011).

51. Kuleshov, M. V. et al. Enrichr: a comprehensive gene set enrichment analysis web server 2016 update. Nucleic Acids Res 44, W90-W97 (2016).

52. Chen, E. Y. et al. Enrichr: interactive and collaborative HTML5 gene list enrichment analysis tool. Bmc Bioinformatics 14, 128-128 (2013).

53. Xie, Z. et al. Gene Set Knowledge Discovery with Enrichr. Curr Protoc 1, e90 (2021).

54. Hollern, D. P., Swiatnicki, M. R. \& Andrechek, E. R. Histological subtypes of mouse mammary tumors reveal conserved relationships to human cancers. Plos Genet 14, e1007135 (2018).

\section{Acknowledgments}

We would like to thank all members of the Hannon and IMAXT labs at the CRUK Cambridge Institute for valuable discussions and advice, in particular Clare Rebbeck for assistance with home office animal licensing. We would also like to thank CRUK Cambridge Institute's core facilities, in particular members of the genomics core for assistance with sequencing library preparation and sequencing, members of the Biological Resource Unit (BRU) for animal husbandry and members of the flow cytometry core for assistance with cell sorting. This work was supported by Cancer Research UK [C14303/A21143, C14303/A19926, C14303/A19927, C9545/A24042]. G.J.H. is a Royal Society Wolfson Research Professor.

\section{Author contributions}

Conceptualization- SAW, KS, IGC, GJH. Data Curation- SAW, IGC, KS. Formal Analysis- SAW, IGC, KS. Funding Acquisition- KS, GJH. Investigation- SAW, IGC, KK. Methodology- SAW, IGC, KS. Project Administration- SAW, KS, IGC, GJH, Software- SAW, KS. Supervision- KS, IGC, GJH. Visualization- SAW, KS, IGC. Writing- Original Draft- SAW, KS, IGC. Writing- reviewing and editing- SAW, KS, IGC, GJH.

\section{Data availability}

Single cell RNA-Seq data are being deposited in the gene expression omnibus (GEO) and will be made available upon publication. All other data are available from the corresponding authors upon reasonable request.

\section{Methods}


bioRxiv preprint doi: https://doi.org/10.1101/2021.12.09.471927; this version posted December 10, 2021. The copyright holder for this preprint (which was not certified by peer review) is the author/funder, who has granted bioRxiv a license to display the preprint in perpetuity. It is made available under aCC-BY 4.0 International license.

\section{Cell lines and culture}

The mouse mammary tumour cell lines 4T1 (ATCC) and D2A1-m2 (kind gifted from Clare Isacke's lab) and the 293FT (Thermo Fisher Scientific) packaging cell line for virus production were cultivated in DMEM high glucose (Gibco), supplemented with 10\% heat-inactivated fetal bovine serum (Gibco) and $50 \mathrm{U} / \mathrm{mL}$ penicillin-streptomycin (Gibco).

\section{Virus production}

The WILD-seq library was packaged using 293FT lentivirus packaging cells. Cells were plated on 15 $\mathrm{cm}$ adherent tissue culture plates (Corning) one day before transfection at a confluency of $\sim 70 \%$. Lentiviral particles were produced by co-transfecting 293FT cells with the transfer plasmid and standard third-generation packaging vectors pMDL $(12.5 \mu \mathrm{g}), \mathrm{CMV}-\mathrm{Rev}(6.25 \mu \mathrm{g})$ and VSV-G $(9 \mu \mathrm{g})$ using the calcium-phosphate transfection method (Invitrogen). The transfection mixture was added to the packaging cells along with $100 \mathrm{mM}$ chloroquine (Sigma-Aldrich). After 16-18 h, media was replaced for fresh growth media. Viral supernatant was collected $48 \mathrm{~h}$ after transfection and filtered through a $45 \mu \mathrm{m}$ filter. The viral supernatant was applied directly to cells or stored at $4^{\circ} \mathrm{C}$ for short-term storage or $-80^{\circ} \mathrm{C}$ for long-term storage. When necessary, virus was concentrated using ultracentrifugation. Lentiviral titre was determined by serial dilutions and measurements of fluorescence via flow cytometry.

\section{WILD-seq library design and cloning}

The pHSW8 lentiviral backbone was constructed using a four-way Gibson Assembly (NEB) by inserting a reverse expression cassette, consisting of a PGK promoter, the zsGreen ORF, a cloning site for highdiversity barcode libraries and a synthetic polyA signal, into an empty pCCL-c-MNDU3-X backbone (\#81071 Addgene). To generate the WILD-seq library, a barcode cassette was introduced at the cloning site within the pHSW8 lentiviral backbone, using PCR (Q5 High-Fidelity DNA Polymerase, NEB) and Gibson Assembly (NEB), such that it is expressed within the 3'UTR of the zsGreen transcript.

\begin{tabular}{|l|l|}
\hline Name & Sequence \\
\hline Assembly_Fwd & 5'-AAACTCTTGAGTGAACTCCAGTGATTTTGAACCAAGCGATTCAAAGTTCT-3' \\
\hline Assembly_Rev & 5'-ccttgccctgaTAACTGGAGGCAGTAATTTACAGCCATGCGCTCGTTTAC-3' \\
\hline BarcodeOligo_Fwd & 5'-TGAACCAAGCGATTCAAAGTTCTATCCGNNNNNNNNNNNNtgcatcggttaaccgatgca-3' \\
\hline BarcodeOligo_Rev & 5'-ATGCGCTCGTTTACTATACGATNNNNNNNNNNNNtgcatcggttaaccgatgca-3' \\
\hline
\end{tabular}

The barcode library was designed by generating $12 \mathrm{nt}$ variable sequences using the $\mathrm{R}$ package DNABarcodes (28052927) and a set Hamming distance of 5. The resulting pool of sequences was then purchased as a custom oligo pool (Twist Bioscience). Reverse complement oligos (BarcodeOligo_Fwd/Rev) each containing a specific PCR handle, a 12-bp variable region and 20-bp constant linker were annealed and amplified by PCR for 20 cycles (using Assembly_Fwd/Rev primers). The amplified barcode library was column purified (Gel extraction kit, Qiagen) and the vector backbone was prepared by digestion with Swal (NEB). WILD-seq barcodes were inserted into the lentiviral vector backbone through Gibson Assembly (NEB), concentrated and transformed into 10b electrocompetent E. coli cells (NEB).

\section{Bottlenecking strategy and characterisation of WILD-seq pools}

4T1 or D2A1-m2 cells were infected with WILD-seq library at low MOI ( 0.2-0.3). Two days after infection, the desired number of zsGreen positive cells, ranging from 10 to 1250 cells, were collected and cultured for two weeks to allow for the pool of clones to stabilize. Different pooling strategies were tested, the ultimate WILD-seq pool was generated from three independent pools each established from 250 sorted cells, maintained separately and mixed in equal proportions immediately prior to injection.

\section{Library complexity analysis}

WILD-seq barcodes of the lentiviral library were amplified using a one-step PCR protocol. 1 ng plasmid was used as template in four separate PCR reactions to account for PCR biases and errors. All reactions were pooled, concentrated and purified on a column and then sequenced on one lane of HiSeq4000. Reads that contained the WILD-seq barcode motif were identified and extracted from the FASTQ files. Detected WILD-seq barcode were filtered based on a $90^{\text {th }}$ percentile cut-off. The resulting whitelist was further filtered for barcodes that contain the common linker region.

\section{Whitelist generation of WILD-seq barcodes}

To generate a comprehensive whitelist of expressed barcodes in each pool, RNA was extracted from WILD-seq transduced cells (High Pure RNA isolation kit, Roche) and reverse transcribed using the Superscript IV reverse transcription kit (Invitrogen) and a target site-specific primer with a unique 
molecular identifier (UMI) and an Illumina sample index. cDNA was amplified by PCR (Q5 High-Fidelity DNA Polymerase, NEB) using primers (RTWhitelist_Fwd/Rev) containing Illumina-compatible adapters. Alternatively, $1 \mu \mathrm{g}$ of gDNA was extracted from WILD-seq transduced cells (Blood\&Cell Culture DNA Kit, Qiagen) and the barcode amplified by PCR using primers containing Illumina-compatible adapters (gDNAWhitelist_Fwd/Rev). PCR products were purified via gel extraction (Qiagen) and quantified by Qubit. The library was sequenced on an Illumina MiSeq with a custom sequencing primer for Read1 (CustomRead1).

\begin{tabular}{|l|l|}
\hline Name & Sequence \\
\hline RT Primer & $\begin{array}{l}\text { 5'-CAAGCAGAAGACGGCATACGAGATNNNNNNGTGACTGGAG } \\
\text { 'TTCAGACGTGTGCTCTTCCGATCTNNNNNNNNCAAGCGATTCAAAGTTCTATCCG-3' }\end{array}$ \\
\hline RTWhitelist_Rev & 5'-CAAGCAGAAGACGGCATACGA-3' \\
\hline RTWhitelist_Fwd & $\begin{array}{l}\text { 5'-AATGATACGGCGACCACCGAGATCTACACCAGCAGTATGCATG } \\
\text { CGCTCGTTTACTATACGAT-3' }\end{array}$ \\
\hline gDNAWhitelist_Fwd & $\begin{array}{l}\text { 5'-AATGATACGGCGACCACCGAGATCTACACCAGCAGTATGCATGC } \\
\text { GCTCGTTTACTATACGAT-3' }\end{array}$ \\
\hline gDNAWhitelist_Rev & $\begin{array}{l}\text { 5'-CAAGCAGAAGACGGCATACGAGATNNNNNNGTGACT } \\
\text { GGAGTTCAGACGTGTGCTCTTCCGATCCAAGCGATTCAAAGTTCTATCCG-3' }\end{array}$ \\
\hline CustomRead1 Primer & 5'-CCAGCAGTATGCATGCGCTCGTTTACTATACGAT-3' \\
\hline
\end{tabular}

Reads from the RT-PCR barcode library that contained the WILD-seq barcode motif were identified and the number of unique UMls supporting each barcode was calculated. If barcode sequences amplified from gDNA were also available an additional filtering step was included and any barcodes not also detected in the gDNA library excluded from the whitelist. Based on UMI counts, the top $90^{\text {th }}$ percentile of detected barcodes were taken and collapsed for PCR and sequencing errors using hierarchical clustering and combining sequences with a Hamming distance less than 5.

\section{Single cell library preparation}

Tumour tissues were collected, minced and dissociated using the gentleMACS Octo Dissociator (Miltenyi Biotec) and the relevant kit (Tumour Dissociation Kit mouse). Tissues were process into single cell suspensions following manufacturer's instructions and filtered through $70 \mu \mathrm{m}$ filters (Miltenyi) to remove any remaining larger particles from single cell suspension after dissociation. The cell suspension was concentrated and filtered again through a $70 \mu \mathrm{m}$ filter. Three million live cells were sorted based on live-dead staining with propidium iodide to remove dead cells and debris, pelleted and resuspended in $1 \mathrm{~mL}$ phosphate-buffered saline with $0.04 \%$ bovine serum albumin (Sigma Aldrich). Cells were counted with a hemocytometer to ensure accurate concentration. The final single cell suspension was diluted as required and sequenced using the Gene Expression 3' v2.1 or v3.1 dual index kit.

\section{Enrichment library preparation}

To enrich for WILD-seq barcodes, the amplified cDNA libraries were further amplified with WILD-seqspecific primers containing Illumina-compatible adapters and sample indices:

\begin{tabular}{|l|l|}
\hline Name & Sequence \\
\hline Enrich_Fwd & 5'-AATGATACGGCGACCACCGAGATCTACACNNNNNNNNNNACACTCTTTCCCTACACGACGCTC-3' \\
\hline Enrich_Rev & $\begin{array}{l}\text { 5'-CAAGCAGAAGACGGCATACGAGATNNNNNNNNNNGTGACTGGAGTTCAGACGTGTGCTCTTC } \\
\text { CGATCTCAGCCATGCGCTCGTTTACTATAC-3' }\end{array}$ \\
\hline \multicolumn{2}{|l|}{ "N" denotes sample indices } \\
\hline
\end{tabular}

$1 \mu \mathrm{L}$ amplified cDNA library was used as template in a 29-cycle PCR reaction using KAPA HiFi HotStart ReadyMix (Roche). To avoid possible PCR-induced library biases, six reactions were run in parallel. All reactions were combined, purified by columns (Gel purification kit, Qiagen) and quantified by Qubit. Gene expression libraries and barcode enrichment libraries were pooled in an approximately 10:1 molar ratio and libraries were sequenced on the NovaSeq platform (Illumina).

\section{Animals and in vivo dosing}

All mouse experiments were performed under the Animals (Scientific Procedures) Act 1986 in accordance with UK Home Office licenses (Project License \# PAD85403A) and approved by the Cancer Research UK (CRUK) Cambridge Institute Animal Welfare and Ethical Review Board. Female six to eight week-old BALB/c were purchased from The Charles River Laboratory. 60,000 tumour cells were 
resuspended in $50 \mu \mathrm{L}$ of a 1:1 mixture of PBS and growth-factor reduced Matrigel (Corning). All orthotopic injections were performed into the fourth mammary gland. Primary tumour volume was measured using the formula $V=0.5\left(L x W^{2}\right)$, in which $W$ is the with and $L$ is length of the primary tumour.

Tumour-bearing mice were treated with either vehicle or with different drugs from seven days post transplantation. All drugs were administered via intraperitoneal injection. For JQ1 treatment, animals were dosed $75 \mathrm{mg} / \mathrm{kg} \mathrm{JQ1}$ (dissolved in DMSO and diluted 1:10 in 10\% $\beta$-cyclodextrin) 5 days/week (5 consecutive days followed by 2 days off) until tumours reached endpoint. For docetaxel treatment, animals were dosed at $12.5 \mathrm{mg} / \mathrm{kg}$ docetaxel (dissolved in 1:1 mixture of ethanol and Kolliphor and diluted 1:4 in saline) 3 times/week, except when L-asparaginase was to be administered concurrently and then the dose was reduced to $10 \mathrm{mg} / \mathrm{kg}$. For L-asparaginase treatment, mice were administered $100 \mu \mathrm{L}$ of $60 \mathrm{U} \mathrm{L}$-asparagine (Abcam) diluted in saline. Vehicle-treated mice were sacrificed 21 days post tumour transplantation and treated animals were sacrificed when tumour volumes reached that of vehicle treated animals at 21 days unless otherwise stated.

\section{ScRNA-seq analysis}

scRNA-seq libraries generated by the 10X Chromium platform were processed using CellRanger version 3.0.1. Reads were aligned to a custom reference genome that was created by adding the sequence of the zsGreen-WILD-seq barcode transgene as a new chromosome to the mm10 mouse genome. The gene expression matrices generated were then analysed with the Seurat $R$ package ${ }^{48}$ using a standard pipeline. Briefly, datasets were first filtered based on the number of unique genes detected per cell (typical accepted range 200-10000 genes) and the percentage of reads that map to the mitochondrial genome (<12\%). Reads which mapped to the zsGreen-WILD-seq barcode transgene were removed from the count matrix to prevent these driving cell clustering. Normalisation was performed using sctransform, including cell cycle regression. Differential abundance of cell subtypes was performed using Milo ${ }^{16}$.

\section{Clonal barcode assignment to single cell data}

Extraction of WILD-seq barcodes from scRNA-seq data: Reads mapping to the zsGreen-WILD-seq barcode transgene and containing the full barcode sequence (20nt constant linker with a $12 \mathrm{nt}$ variable region on either side) were extracted from the BAM file produced by Cell Ranger and mapped using Bowtie to a whitelist of barcodes expressed in the WILD-seq cell pool. A WILD-seq clonal barcode was assigned to a cell if there were at least 2 independent reads which matched the barcode to the cell and more than $50 \%$ of barcode mapped reads from the cell supported the assignment.

Extraction of WILD-seq barcodes from PCR enrichment data: Reads from the PCR barcode enrichment were processed separately using the UMI-tools to extract 10X cell barcodes and UMls from the raw read files. The sequence corresponding to the full barcode sequence (20nt constant linker with a $12 \mathrm{nt}$ variable region on either side) was extracted from each read and then mapped to the WILD-seq clonal barcode whitelist using Bowtie. A WILD-seq clonal barcode was assigned to a cell if there were at least 10 UMls which matched the barcode to the cell and at least twice as many UMls supporting this assignment compared to the next best.

WILD-seq barcode assignment: The WILD-seq clonal barcode assignment from these 2 pipelines was then compared. If the assignment from the transcriptomic analysis and the PCR enrichment analysis were in agreement the barcode was assigned. On the rare occasion the assignment didn't match a clonal barcode was not assigned. If a cell was assigned a WILD-seq barcode by only one method, a further more stringent filtering step was included. For WILD-seq barcodes assigned only from the 10X scRNA-seq dataset but not the PCR-enrichment, the minimum number of UMls required to support the assignment was increased to 5 and for WILD-seq barcodes assigned only from the PCR-enrichment but not the 10X scRNA-seq dataset, the minimum number of UMls required to support the assignment was increased to 30 .

\section{Differential gene expression}

Differential gene expression was determined using the FindMarkers function in Seurat with a Wilcoxon rank sum test to identify differentially expressed genes. For differential expression of groups of genes, we used the AUCell R package ${ }^{14}$ which enables analysis of the relative expression of a gene set (i.e. gene signature or pathway) across all the cells in single-cell RNA-seq data using the "Area Under the Curve" (AUC) to calculate the enrichment of the input geneset within the expressed genes for each cell. An AUCell score was calculated for each tumour cell for every gene set in the MSigDB C2 collection ${ }^{49,50}$ 
that contained more than 20 genes with detectable expression in our data. AUCell scores were compared across clones or conditions using a Wilcoxon rank sum test and p-values were adjusted for multiple comparison using the Benjamini-Hochberg correction method.

To generate baseline transcriptomic signatures for each clone in vehicle-treated tumours, comparisons were made between the clone of interest and all assigned tumour cells from the same sample (in the case of D2A1 tumours) or the same experiment (in the case of 4T1 tumours). Samples/experiments were included if they contained at least 20 cells assigned to the clone of interest. To define consistently enriched/depleted signatures, $p$-values from comparisons within each sample/experiment were combined using the Fisher's method.

\section{Patient data analysis}

Microarray gene expression data was downloaded from GSE28844 ${ }^{38}$. A single probe for each gene was selected based on the highest median expression. Gene set expression per patient sample was calculated using GSVA ${ }^{39}$.

\section{Description of supplementary tables}

\section{Supplementary Table 1. Overview of single cell RNA-seq samples generated.}

Supplementary Table 2. Number and proportion of tumour cells assigned to each clonal barcode for all 4T1 WILD-seq sample.

\section{Supplementary Table 3. Number and proportion of tumour cells assigned to each clonal barcode} for all D2A1 WILD-seq sample.

Supplementary Table 4. 4T1 WILD-seq baseline gene enrichment signatures for major clones. Differential gene expression analysis was performed for each clone by comparing cells from a clonal lineage of interest to all assigned tumour cells within the same experiment. Only vehicle-treated samples were included in the analysis. Experiments were included in the analysis if they contained at least 20 cells assigned to the clone and clones were analysed if they were represented by at least 20 cells in at least 3 of the 4 experiments. Differential gene expression was performed using Seurat FindMarkers function and Wilcoxon Rank Sum test. Fisher's method was used to combine p-values from separate experiments. Analysis for each clone is provided as a separate tab.

Supplementary Table 5. 4T1 WILD-seq baseline gene set enrichment signatures for major clones. Differential gene set expression analysis was performed for each clone by comparing cells from a clonal lineage of interest to all assigned tumour cells within the same experiment. All gene sets from the Molecular Signatures Database C2 curated gene set collection were included in the analysis that contained more than 20 genes detectable in our single cell data. Only vehicle-treated samples were included in the analysis. Experiments were included in the analysis if they contained at least 20 cells assigned to the clone and clones were analysed if they were represented by at least 20 cells in at least 3 of the 4 experiments. Gene set expression analysis was performed using AUCell and differential expression was calculated using Wilcoxon Rank Sum test. Tables show median AUCell score per experiment for each gene set, enrichment in AUCell score relative to all assigned tumour cells within the same experiment $\left(\log _{2}\right.$ (median AUCell score clone of interest/median AUCell score all clones)) and adjusted $p$-value from Wilcoxon Rank Sum test of AUCell scores from clone of interest vs AUCell scores from all assigned tumours cells from the same experiment. Fisher's method was used to combine $p$ values from separate experiments. Analysis for each clone is provided as a separate tab. A final tab 'Data_for_Fig1h' provides the matrix of AUCell enrichment values used for the heatmap plotted in figure 1h compiled from individual analyses.

Supplementary Table 6. Differential expression analysis JQ1 vs Vehicle. Differential gene expression analysis was performed by comparing cells from the same clonal lineage treated with JQ1 or vehicle within the same experiment. Five clones were included in the analysis (clones 350, 473, 537, 606 and 684) for which there were at least 20 cells per condition across both experiments. Fisher's method was used to combine $p$-values from different clones within the same experiment. Gene level differential expression was performed using Seurat FindMarkers function and Wilcoxon Rank Sum test. These data are provided under the 'FindMarkers_JQ1vsVeh' tab. Gene set level differential expression was performed using AUCell and differential expression was calculated using Wilcoxon Rank Sum test. These data are provided under the 'AUCell_JQ1vsVeh' tab. The 'Median_norm_AUCell_Scores' tab provides a summary of the median normalised AUCell scores for each clone, condition and experiment used in the preparation of figure $2 \mathrm{e}$. Normalisation to enable comparison across separate experiments 
bioRxiv preprint doi: https://doi.org/10.1101/2021.12.09.471927; this version posted December 10, 2021. The copyright holder for this preprint (which was not certified by peer review) is the author/funder, who has granted bioRxiv a license to display the preprint in perpetuity. It is made available under aCC-BY 4.0 International license.

was performed by dividing by the median AUCell score for all vehicle-treated tumour cells assigned to any clonal lineage from the same experiment.

Supplementary Table 7. Correlation of clonal gene expression with JQ1 response. To determine genes and gene sets whose expression correlates with JQ1 response, the correlation between baseline gene and geneset enrichment values for the major clones as defined in supplementary tables 4 and 5 and the log fold change in clonal abundance between JQ1 and vehicle-treated samples was calculated using the Pearson correlation test. The Pearson correlation coefficient is provided for each gene and gene set.

Supplementary Table 8. Correlation of clonal gene expression with docetaxel response. To determine genes and gene sets whose expression correlates with docetaxel response, the correlation between baseline gene and geneset enrichment values for the major clones as defined in supplementary tables 4 and 5 and the log fold change in clonal abundance between JQ1 and vehicletreated samples was calculated using the Pearson correlation test. The Pearson correlation coefficient is provided for each gene and gene set.

Supplementary Table 9. D2A1 WILD-seq baseline gene enrichment signatures for major clones. Differential gene expression analysis was performed for each clone by comparing cells from a clonal lineage of interest to all assigned tumour cells within the same sample. Only vehicle-treated samples were included in the analysis. Clones were included in the analysis if there were at least 20 cells assigned to that clone in all three vehicle samples (DV1, DV2 and DV3). Differential gene expression was performed using Seurat FindMarkers function and Wilcoxon Rank Sum test. Fisher's method was used to combine $p$-values from separate samples. Analysis for each clone is provided as a separate tab. In addition, analysis is included for the combined resistant clones 7511197 and 1240. Due to their low representation in vehicle-treated samples cells assigned to these clones from all three vehicletreated samples were combined for gene expression analysis and compared to all assigned tumour cells from the three samples.

Supplementary Table 10. D2A1 WILD-seq baseline gene set enrichment signatures for major clones. Differential gene set expression analysis was performed for each clone by comparing cells from a clonal lineage of interest to all assigned tumour cells within the same sample. All gene sets from the Molecular Signatures Database C2 curated gene set collection were included in the analysis that contained more than 20 genes detectable in our single cell data. Only vehicle-treated samples were included in the analysis. Clones were included in the analysis if there were at least 20 cells assigned to that clone in all three vehicle samples (DV1, DV2 and DV3). Gene set expression analysis was performed using AUCell and differential expression was calculated using Wilcoxon Rank Sum test. Tables show median AUCell score per sample for each gene set, enrichment in AUCell score relative to all assigned tumour cells within the same experiment $\left(\log _{2}\right.$ (median AUCell score clone of interest/median AUCell score all clones)) and adjusted p-value from Wilcoxon Rank Sum test of AUCell scores from clone of interest vs AUCell scores from all assigned tumours cells from the same sample. Fisher's method was used to combine p-values from separate samples. Analysis for each clone is provided as a separate tab. In addition, analysis is included for the combined resistant clones 7511197 and 1240. Due to their low representation in vehicle-treated samples cells assigned to these clones from all three vehicle-treated samples were combined for gene expression analysis and compared to all assigned tumour cells from the three samples. The tab 'Data for Fig4e and $4 \mathrm{f}$ ' provides the matrix of median AUCell scores used for the heatmap plotted in figure $4 \mathrm{e}$ compiled from individual analyses. The tab 'Data for Fig4h' provides median AUCell scores per sample for clones of interest for all samples and conditions where at least 20 cells per clone were present. Selected data from this table was plotted in figure $4 \mathrm{~h}$.

Supplementary Table 11. Comparison of differential gene expression analysis in bulk tumour cells and intra-clonal changes in gene expression. For each treatment condition (docetaxel/D2A1, docetaxel/4T1 and JQ1/4T1) differential expression analysis was performed between barcoded tumour cells from drug-treated and vehicle-treated animals from the same experiment. Analysis was performed either by using cells from a single clonal lineage (analysis by clone) or all barcoded tumour cells irrespective of clonal lineage (bulk tumour cell analysis). Differential gene expression was performed using Seurat FindMarkers function and Wilcoxon Rank Sum test. Log $_{2}$ fold change and adjusted $p$ value are provided for each comparison. For the analysis by clone, the mean logFC of all individual clonal comparisons is given (mean.logFC.clonal) and Fisher's method was used to combine p-values (fisher.combined.pvalue.clonal). Genes were classified as significantly changed in clonal analysis only, bulk analysis only or both analysis methods based on significance cutoffs of $p$-value $<0.05$ and logFC $<-0.2$ or $>0.2$. Genes identified as significantly changing by one method only met neither logFC nor $p-$ 
bioRxiv preprint doi: https://doi.org/10.1101/2021.12.09.471927; this version posted December 10, 2021. The copyright holder for this preprint (which was not certified by peer review) is the author/funder, who has granted bioRxiv a license to display the preprint in perpetuity. It is made available under aCC-BY 4.0 International license.

value cutoffs in the alternative method. For analysis of WILD-seq 4T1 data, analysis was performed separately for the 2 experiments and genes had to meet significance cutoffs in both experiments. Supplementary Table 12. Overlap of docetaxel resistance markers in 4T1 and D2A1 cell lines. $4 \mathrm{~T} 1$ resistance genes were defined as those that were significantly enriched in resistant clone 679 but not in sensitive clone $238(p<0.05)$. D2A1 resistance genes were defined as those that were significantly enriched in combined resistant clones 1240, 751 and 1197 but not in sensitive clones 118,2874 or $1072(p<0.05)$. Overlap of these lists revealed 47 common genes. These are listed along with their human orthologs. 
bioRxiv preprint doi: https://doi.org/10.1101/2021.12.09.471927; this version posted December 10, 2021. The copyright holder for this preprint (which was not certified by peer review) is the author/funder, who has granted bioRxiv a license to display the preprint in

a perpetuity. It is made available under aCC-BY 4.0 International license.

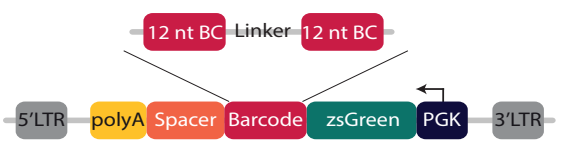

C

\section{in vitro 4T1}

WILD-seq cell pool

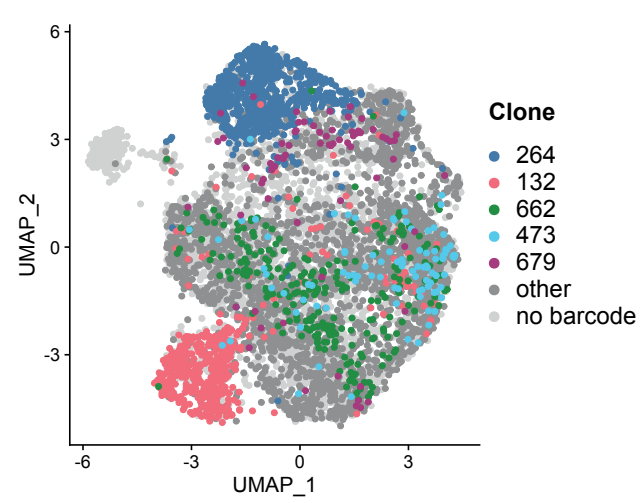

e

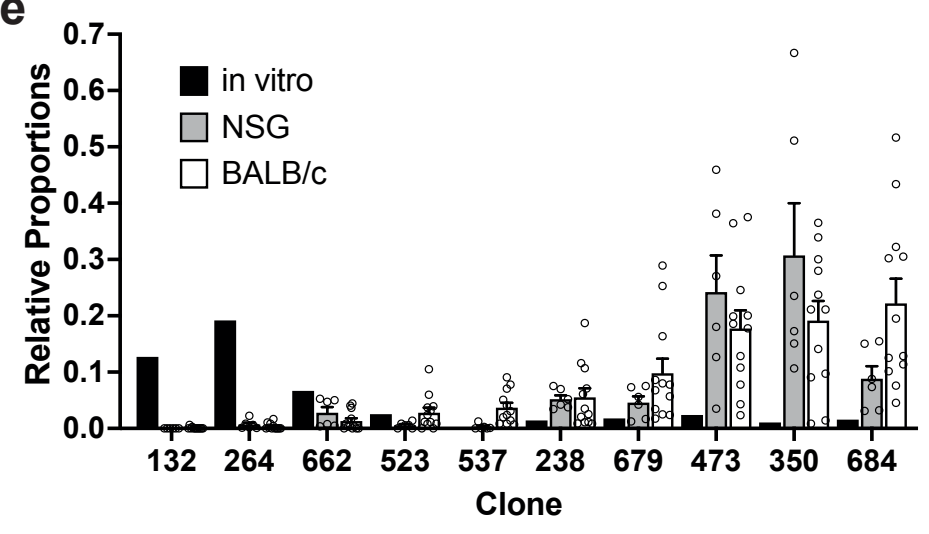

b

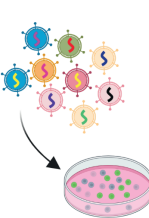

Lentiviral
infection
of WILDseq of WILDseq
barcodes

d

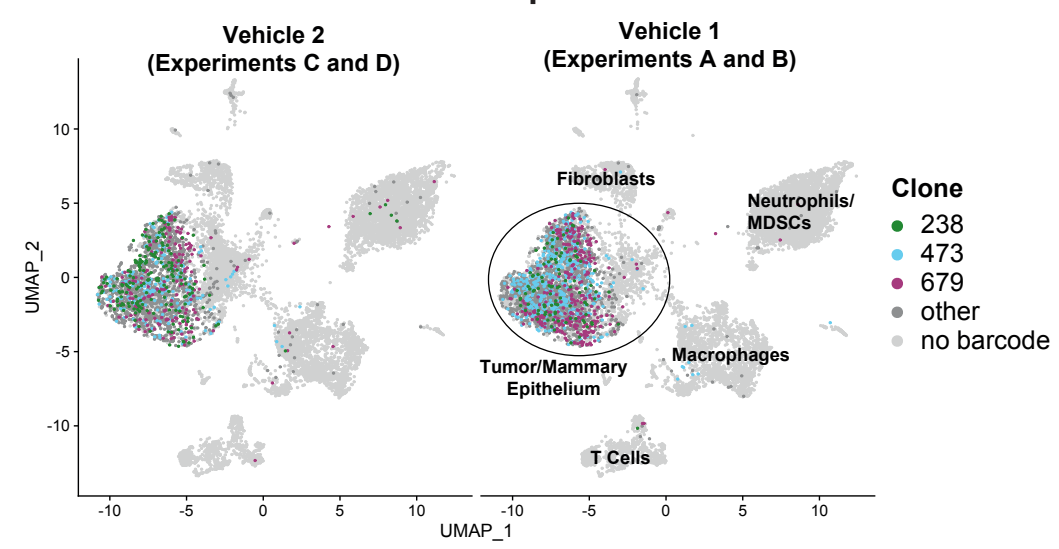

g

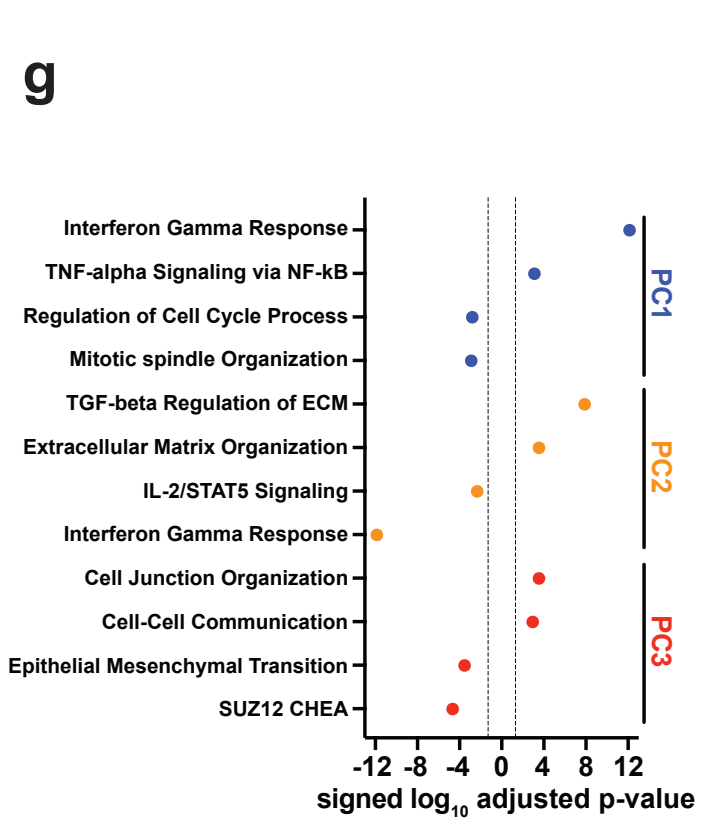

h

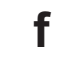

f

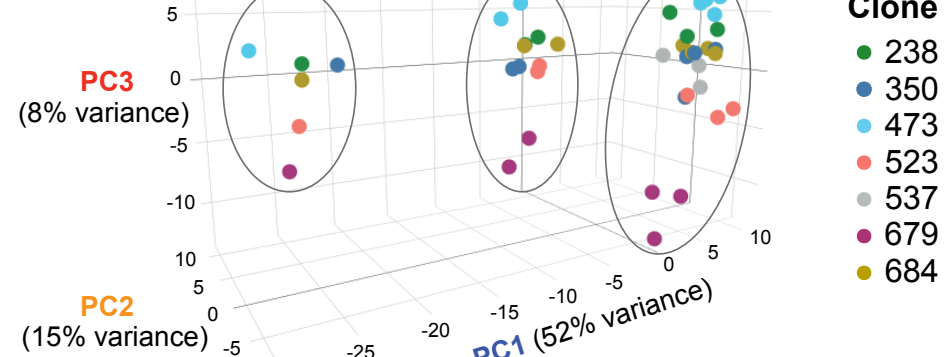

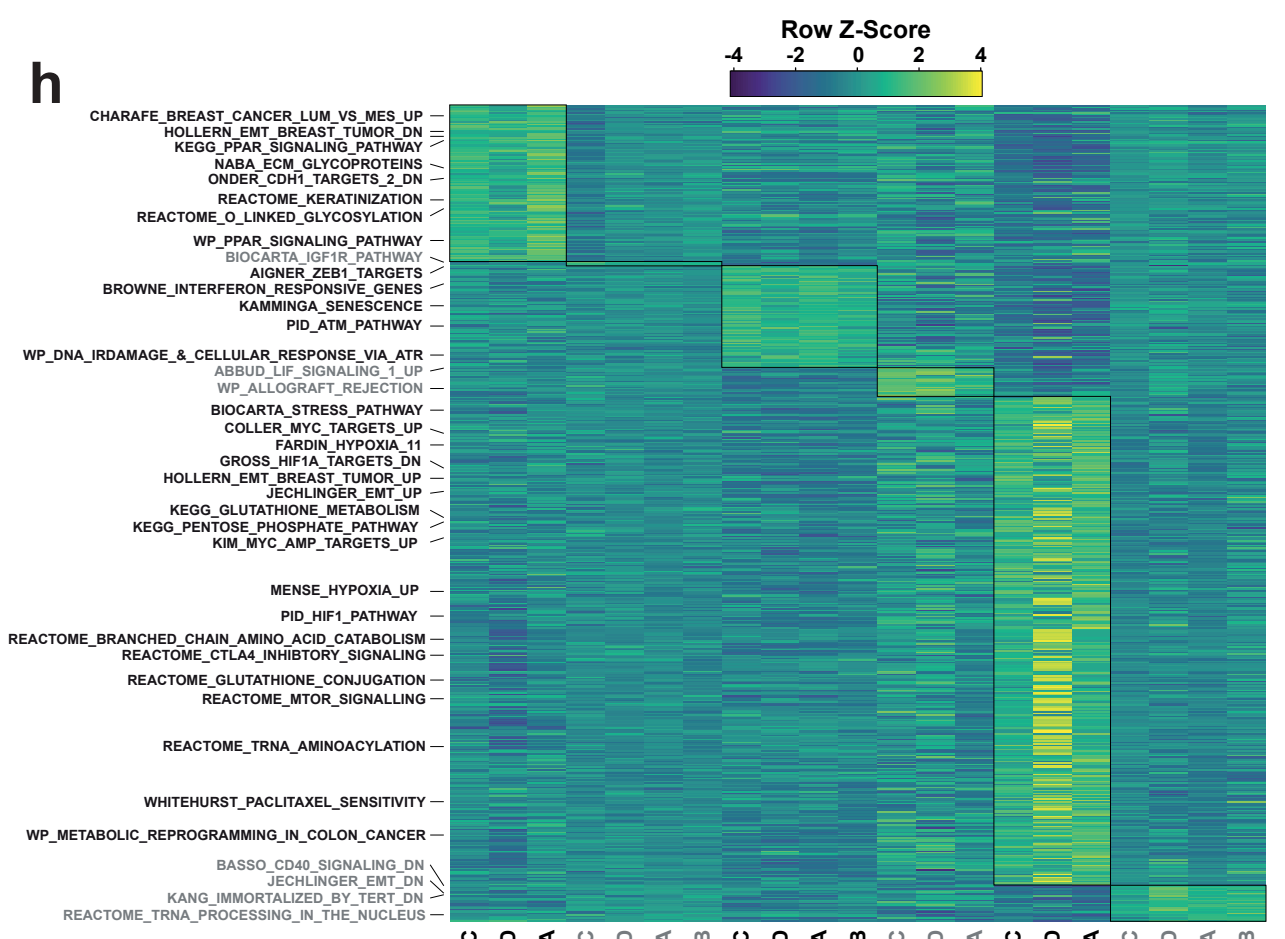

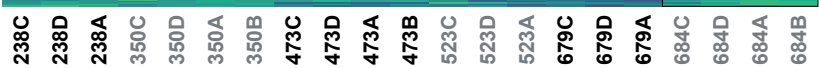


Figure 1. Establishment of an expressed barcode system to simultaneously detect clonal lineage and gene expression from single cells in vivo.

a. Lentiviral construct design. A PGK promoter drives expression of a transcript encoding zsGreen harbouring a WILD-seq barcode sequence in the 3'UTR. A spacer sequence and polyadenylation signal ensure that that the barcode is detectable as part of a standard oligo dT single cell RNA library preparation and sequencing pipeline. The barcode cassette comprises 2 distinct 12 nucleotide barcode sequences separated by a constant 20 nucleotide linker region. The library of barcode sequences was designed with Hamming distance 5 to allow for sequencing error correction. b. Schematic of WILDseq method. Tumour cells are infected with the WILD-seq lentiviral library and an appropriate size population of zsGreen positive cells isolated, each of which will express a single unique WILD-seq barcode. This WILD-seq barcoded, heterogenous cell pool is then subjected to an intervention of interest (such as in vivo treatment of the implanted pool with a therapeutic agent) and subsequently analysed by single cell RNA sequencing using the 10X Genomics platform. An additional PCR amplification step is included that specifically enriches for the barcode sequence to increase the number of cells to which a WILD-seq barcode can be conclusively assigned. c. scRNA-seq of in vitro 4T1 WILD-seq cell pool. UMAP plot of in vitro cultured 4T1 WILD-seq cells. Cells for which a WILD-seq clonal barcode is identified are shown as dark grey or coloured spots. Cells which belong to five selected clonal lineages are highlighted. d. scRNA-seq of 4T1 WILD-seq tumours. UMAP plots of vehicletreated $4 T 1$ WILD-seq tumours generated by injecting the $4 T 1$ WILD-seq pool into the mammary fatpad of BALB/c mice. Four independent experiments were performed each involving injection into 3 separate host animals. Six animals from experiments A and B received vehicle $1(10 \%$ DMSO, $0.9 \% \beta-$ cyclodextrin) and six animals from experiments $C$ and $D$ received vehicle $2(12.5 \%$ ethanol, $12.5 \%$ Kolliphor). e. Clonal representation. Proportion of tumour cells assigned to each clonal lineage based on the WILD-seq barcode ( $n=1$ for in vitro cultured cells, $n=6$ for tumours from NSG mice, $n=12$ for vehicle-treated tumours from BALB/c mice). Selected clones from the most abundant lineages are plotted. Data represents mean \pm SEM. f. Principal component analysis of clonal transcriptomes. Pseudo-bulk analysis was performed by summing counts for all tumour cells expressing the same WILD-seq clonal barcode within an independent experiment. For in vivo tumour samples each point represents the combined cells from 3 animals. Principal component analysis of normalized pseudo-bulk count data showed separation of samples by origin with PC1 and PC2 and separation by clonality with PC3. g. Transcriptomic programs associated with principal components. The top/bottom 50 gene loadings of PC1, PC2 and PC 3 were analysed using Enrichr $r^{51-53}$. h. Clonal transcriptomic signatures from vehicle-treated BALB/c tumours. An AUCell score ${ }^{14}$ enrichment was calculated for each clone and for each experiment by comparing cells of a specific clonal lineage of interest to all assigned tumour cells within the same experiment. All gene sets which showed consistent and statistically significant enrichment in one of the six most abundant clones across experiments are illustrated. 
bioRxiv preprint doi: https://doi.org/10.1101/2021.12.09.471927; this version posted December 10, 2021. The copyright holder for this preprint (which was not certified by peer review) is the author/funder, who has granted bioRxiv a license to display the preprint in

a
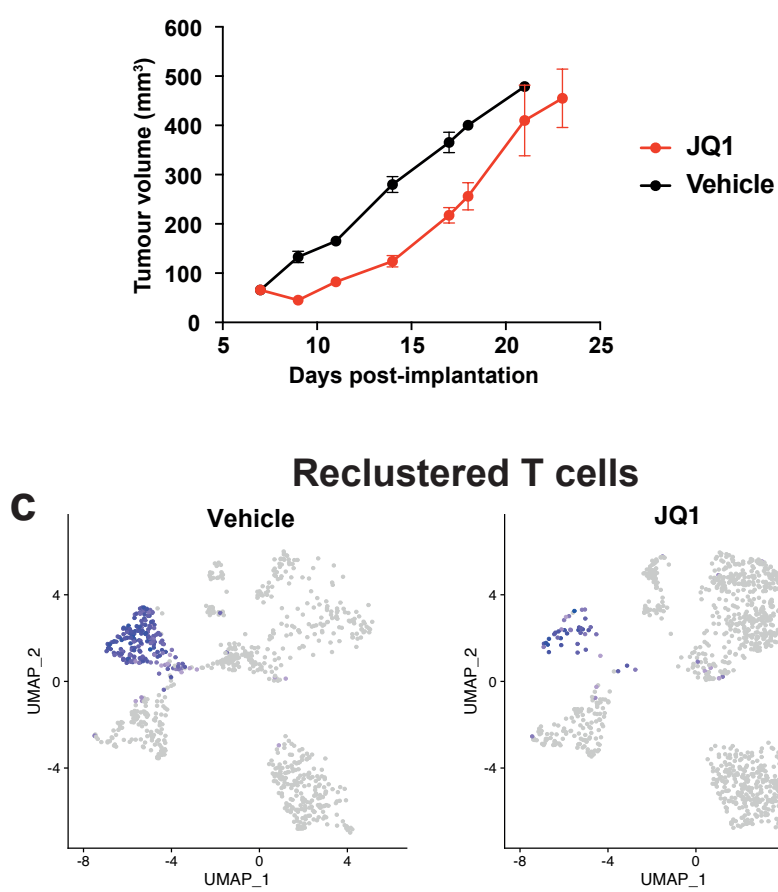

Differential expression JQ1 vs Vehicle Experiment A

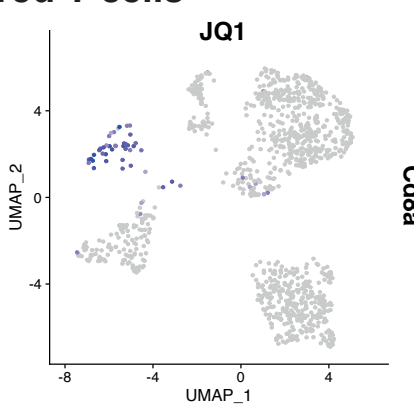
xperiment B perpetuity. It is made available

brenter

JQ1
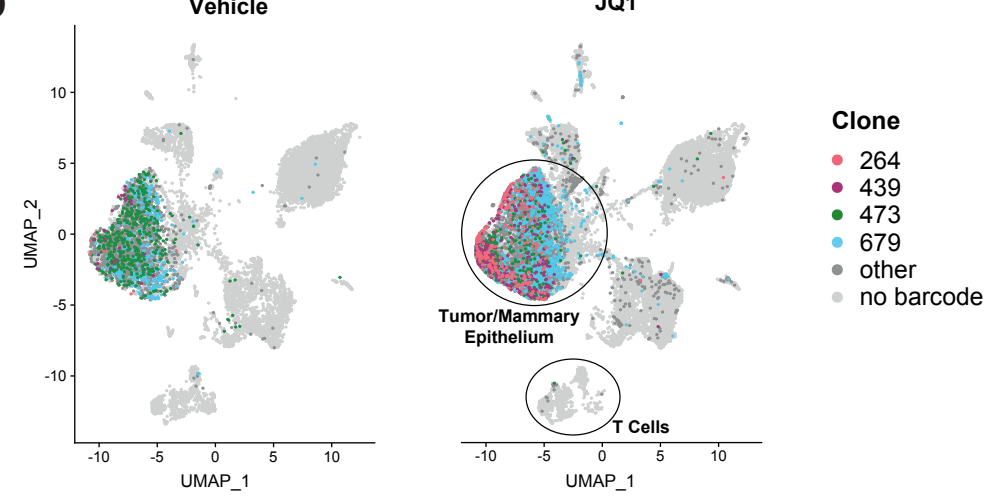

d

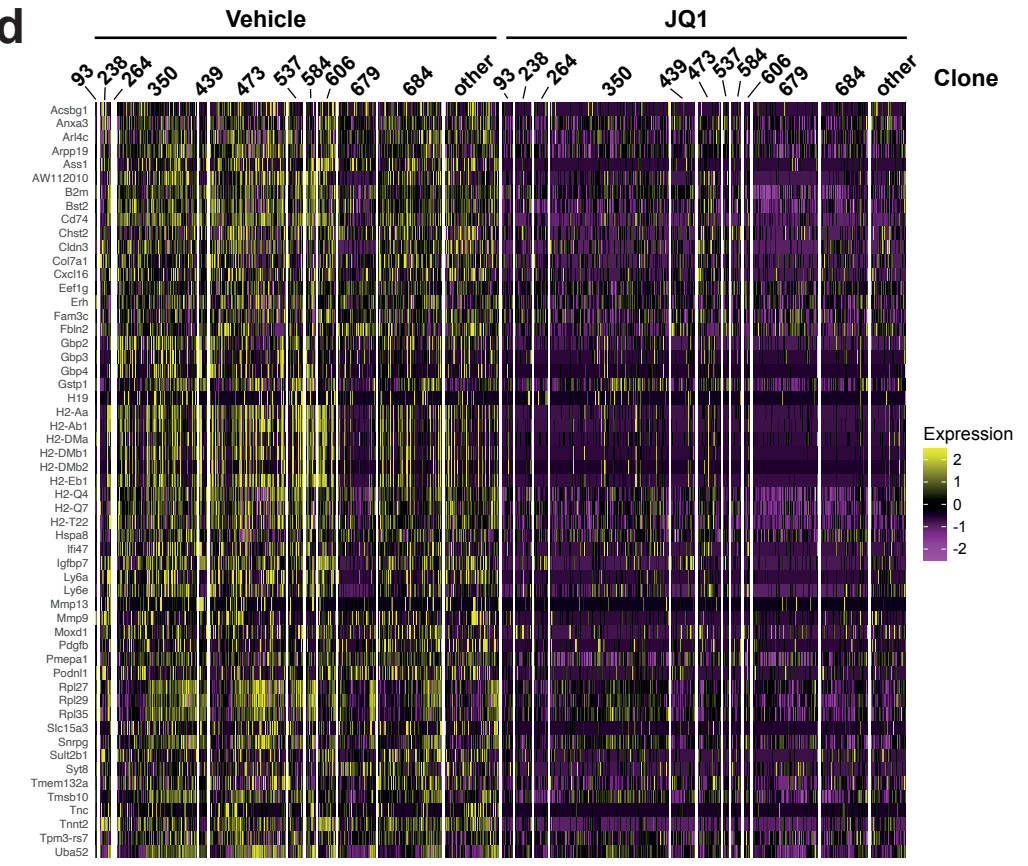

e

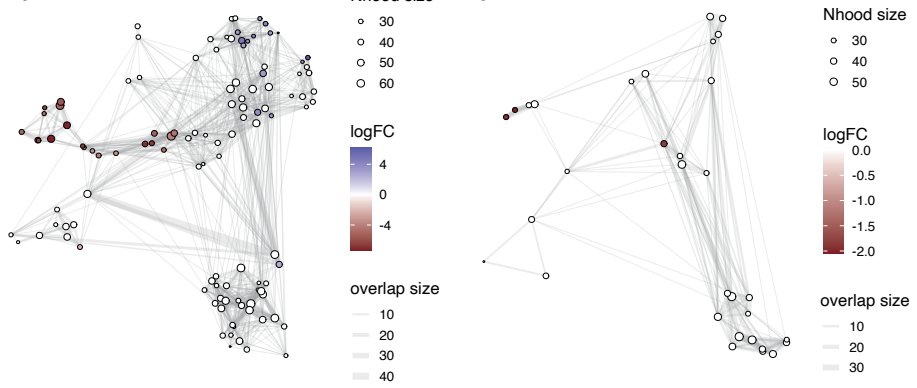

Differential expression JQ1 vs Vehicle
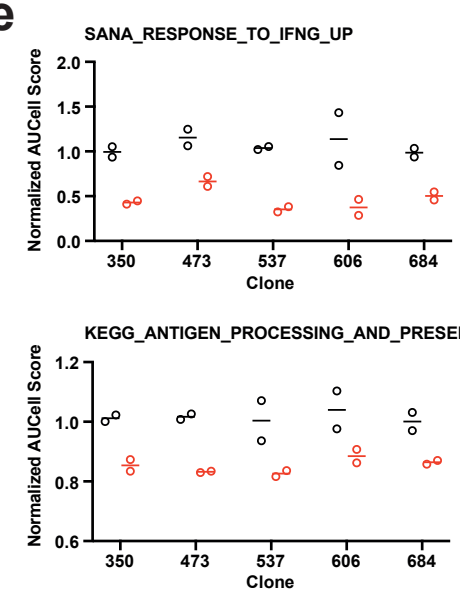

g
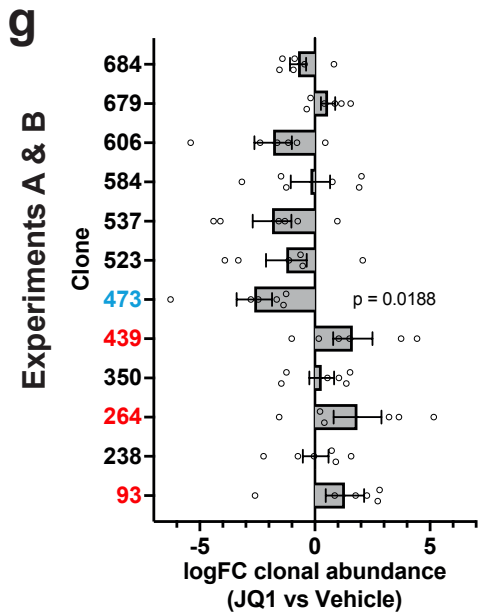
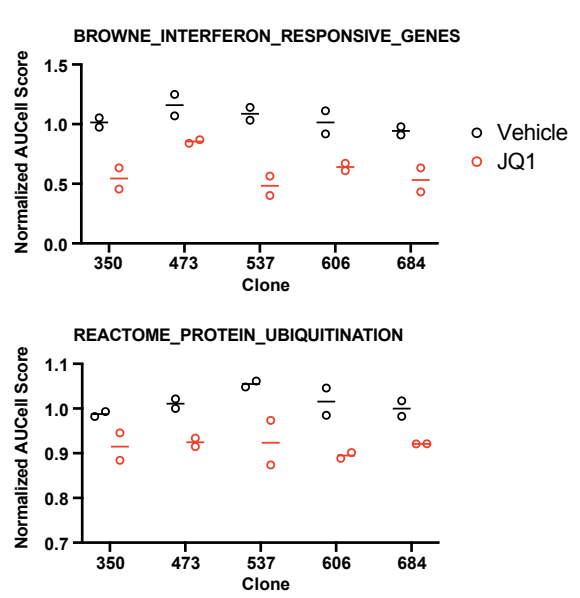

h

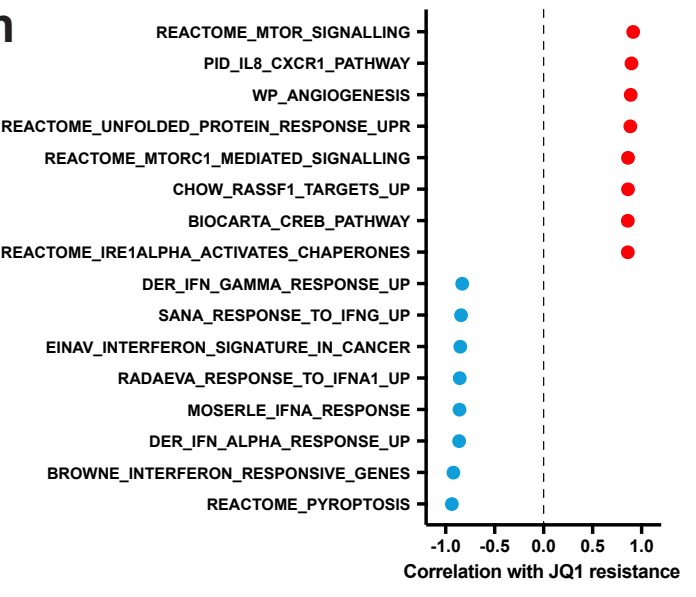

$\mathbf{f}$

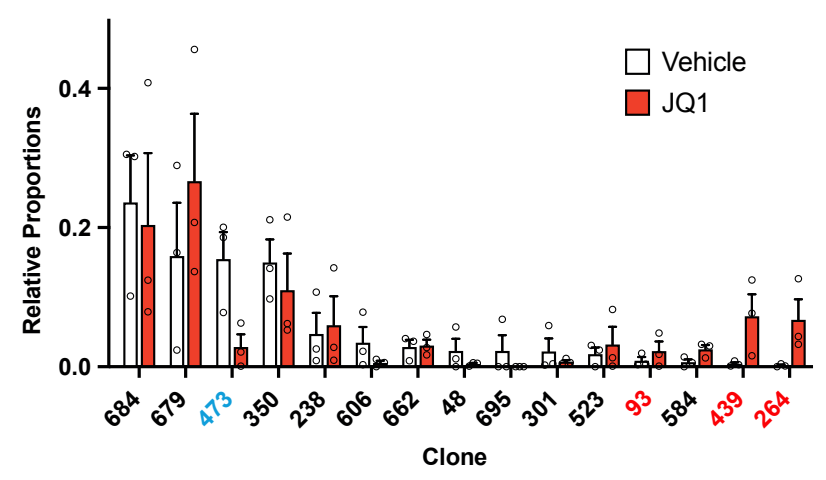


Figure 2. Simultaneous detection of changes in clonal abundance, gene expression, and tumour microenvironment in response to BET bromodomain inhibition with WILD-seq. a. Tumour growth curves with JQ1 treatment. 4T1 WILD-seq tumours were treated with the BET bromodomain inhibitor JQ1 or vehicle from 7 days post-implantation until endpoint $(n=4$ mice per condition). Data represents mean \pm SEM. b. scRNA-seq of JQ1-treated 4T1 WILD-seq tumours. UMAP plots of vehicle- or JQ1-treated 4T1 WILD-seq tumours. Combined cells from 2 independent experiments, each with 3 mice per condition are shown. Cells for which a WILD-seq clonal barcode is identified are shown as dark grey or coloured spots. Cells which belong to four selected clonal lineages are highlighted. c. JQ1-treatment results in a reduction in Cd8+ tumour-associated T-cells. Cells belonging to the Tcell compartment were computationally extracted from the single cell data and reclustered. Upper panels show combined UMAP plots from experiments $A$ and $B$ with $C d 8 a$ expression per cell illustrated enabling identification of the $\mathrm{Cd} 8+\mathrm{T}$ cell cluster. Lower panels show neighborhood graphs of the results from differential abundance testing using Milo $^{16}$. Coloured nodes represent neighbourhoods with significantly different cell numbers between conditions (FDR $<0.05$ ) and the layout of nodes is determined by the position of the neighborhood index cell in the UMAP panel above. Experiments $A$ and $B$ were analysed separately due to differences in cell numbers. d. Differential gene expression between JQ1- and vehicle-treated tumour cells. Single cell heatmap of expression for genes which are significantly and consistently down-regulated across clonal lineages (combined fisher $p$-value < 0.05 and mean $\operatorname{logFC}<-0.2$ for both experiments). 1600 cells are represented (400 per experiment/condition), grouped according to their clonal lineage. e. Differential gene set expression between JQ1 and vehicle-treated tumour cells. Median AUCell score per experiment/condition for selected gene sets. The 5 clonal lineages with the highest representation across experiments are shown. f. Clonal representation. Proportion of tumour cells assigned to each clonal lineage in experiment $A$ based on the WILD-seq barcode ( $n=3$ tumours per condition). Clones which make up at least $2 \%$ of the assigned tumour cells under at least one condition are plotted. The most sensitive clone 473 is highlighted in blue and the most resistant clones 93, 439, 264 are highlighted in red. Data represents mean \pm SEM. g. Clonal response to JQ1-treatment. $\log _{2}$ fold change in clonal proportions upon JQ1 treatment across experiments A and B. Fold change was calculated by comparing each JQ1treated sample with the mean of the 3 corresponding vehicle-treated samples from the same experiment. p-value calculated by one-sample t-test vs a theoretical mean of 0 . Data represents mean \pm SEM. $h$. and i. Correlation of JQ1-response with baseline clonal transcriptomic signatures. Clonal gene set enrichment scores for vehicle-treated tumours were calculated by comparing cells of a specific clonal lineage of interest to all assigned tumour cells within the same experiment. Correlation between these scores and JQ1-treatment response (mean $\log _{2}$ fold change clonal proportion JQ1 vs vehicle) was then calculated for each gene set. Selected gene sets with the highest positive or negative correlation values (Pearson correlation test) are shown. A positive correlation indicates a higher expression in resistant clones, whereas a negative correlation indicates a higher expression in sensitive clones. Resistant clonal lineages identified by barcodes 93,264 and 439 were combined for the purpose of this analysis to have enough cells for analysis within the vehicle-treated samples. 
bioRxiv preprint doi: https://doi.org/10.1101/2021.12.09.471927; this version posted December 10, 2021. The copyright holder for this

preprint (which was not certified by peer review) is the author/funder, who has granted bioRxiv a license to display the preprint in perpetuity. It is made $\mathrm{B}$ Jailable under aCC-BY 4.0 International license.
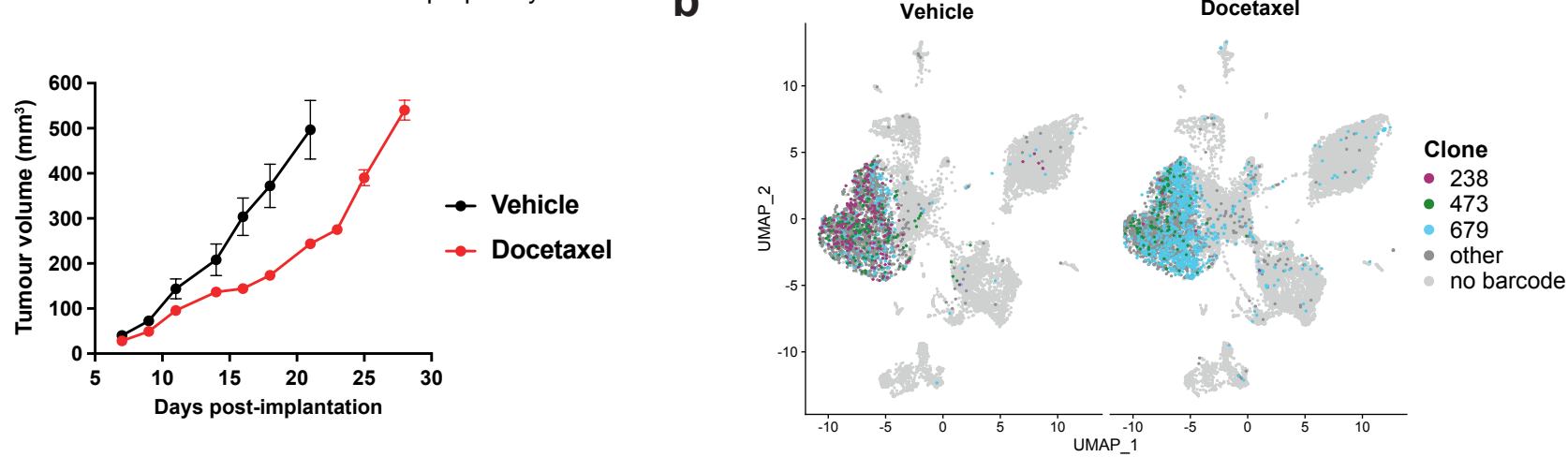

C

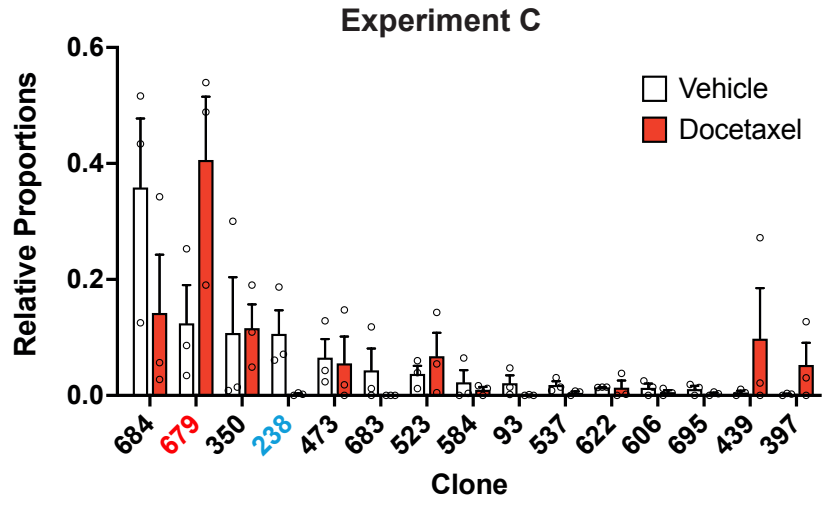

e

HONMA_DOCETAXEL_RESISTANCE
KEGG_INSULIN_SIGNALING_PATHWAY
REACTOME_RESPONSE_OF_EIF2AK4_GCN2_TO_AMINO_ACID_DEFICIENCY REACTOME_HATS_ACETYLATE_HISTONES REACTOME_GLYCOGEN_METABOLISM BILANGES_SERUM_AND_RAPAMYCIN_SENSITIVE_GENES

LI_CISPLATIN_RESISTANCE_UP KEGG_GLUTATHIONE_METABOLISM KEGG_CELL_ADHESION_MOLECULES_CAMS PID_DELTA_NP63_PATHWAY AIGNER_ZEB1_TARGETS

BOSCO_EPITHELIAL_DIFFERENTIATION_MODULE HOLLERN_EMT_BREAST_TUMOR_DN CHARAFE_BREAST_CANCER_LUMINAL_VS_MESENCHYMAL_UP HOLLEMAN_ASPARAGINASE_RESISTANCE_B_ALL_UP ONDER_CDH1_TARGETS_2_DN

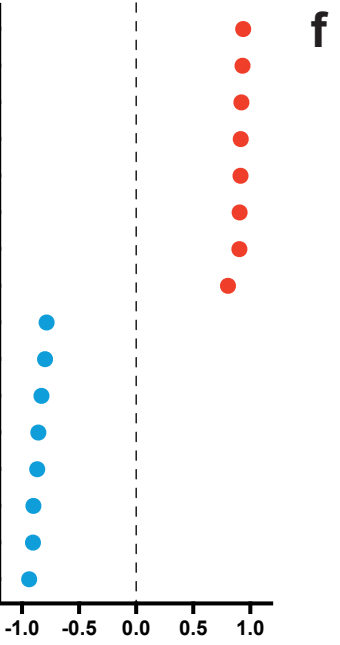

Correlation with Docetaxel Resistance d

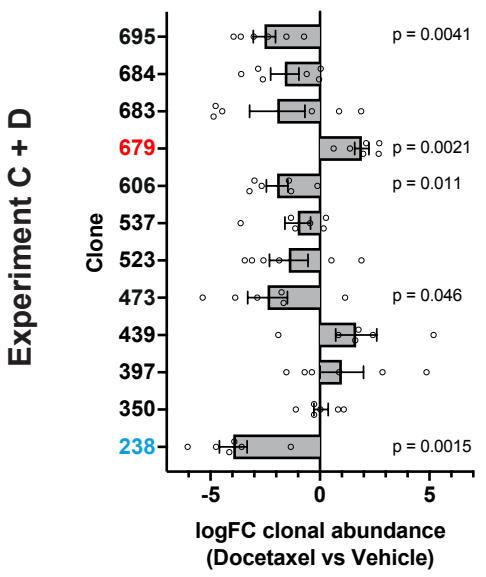

CHARAFE_BREAST_CANCER

HONMA_DOCETAXEL

LUMINAL_VS_MESENCHYMAL_UP

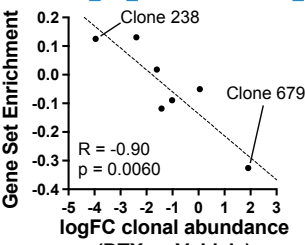

(DTX vs Vehicle)

HOLLEMAN_ASPARAGINASE RESISTANCE_B_B_ALL_UP

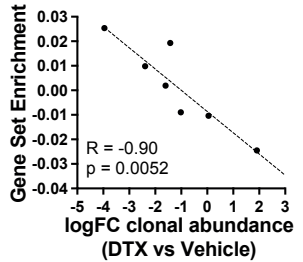
RESISTANCE

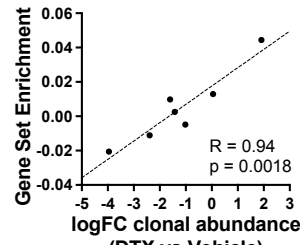

(DTX vs Vehicle)

REACTOME_GLYCOGEN METABOLISM

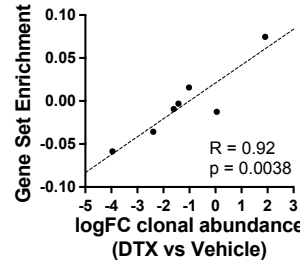


bioRxiv preprint doi: https://doi.org/10.1101/2021.12.09.471927; this version posted December 10, 2021. The copyright holder for this preprint (which was not certified by peer review) is the author/funder, who has granted bioRxiv a license to display the preprint in perpetuity. It is made available under aCC-BY 4.0 International license.

Figure 3. Clonal transcriptomic correlates of response and resistance to taxane chemotherapy in the 4T1 mammary carcinoma model. a. Tumour growth curves with docetaxel treatment. 4T1 WILD-seq tumours were treated with docetaxel or vehicle (12.5\% ethanol, $12.5 \%$ Kolliphor) from 7 days post-implantation for 2 weeks ( $n=5$ mice per condition). Dosing regimen was $12.5 \mathrm{mg} / \mathrm{Kg}$ docetaxel three times per week. Data represents mean \pm SEM. b. scRNA-seq of docetaxel-treated 4T1 WILDseq tumours. UMAP plots of vehicle- or docetaxel-treated 4T1 WILD-seq tumours. Combined cells from 2 independent experiments, each with 3 mice per condition are shown. Cells for which a WILDseq clonal barcode is identified are shown as dark grey or coloured spots. Cells which belong to three selected clonal lineages are highlighted. c. Clonal representation. Proportion of tumour cells assigned to each clonal lineage in experiment $C$ based on the WILD-seq barcode ( $n=3$ tumours per condition). Clones which make up at least $2 \%$ of the assigned tumour cells under at least one condition are plotted. The most sensitive clone 238 is highlighted in blue and the most resistant clone 679 is highlighted in red. Data represents mean \pm SEM. d. Clonal response to docetaxel-treatment. $\log _{2}$ fold change in clonal proportions upon docetaxel treatment across experiments $C$ and $D$. Fold change was calculated by comparing each docetaxel-treated sample with the mean of the 3 corresponding vehicle-treated samples from the same experiment. $p$-values calculated by one-sample t-test vs a theoretical mean of 0 . Data represents mean \pm SEM. e. and $f$. Correlation of docetaxel-response with baseline clonal transcriptomic signatures. Clonal gene set enrichment scores for vehicle-treated tumours were calculated by comparing cells of a specific clonal lineage of interest to all assigned tumour cells within the same experiment. Correlation between these scores and docetaxel-treatment response (mean $\log _{2}$ fold change clonal proportion docetaxel vs vehicle) was then calculated for each gene set. Selected gene sets with the highest positive or negative correlation values (Pearson correlation test) are shown. A positive correlation indicates a higher expression in resistant clones, whereas a negative correlation indicates a higher expression in sensitive clones. 
bioRxiv preprint doi: https://doi.org/10.1101/2021.12.09.471927; this version posted December 10, 2021. The copyright holder for this preprint (which was not certified by peer review) is the author/funder, who has granted bioRxiv a license to display the preprint in perpetuity. It is made available under aCC-BY 4.0 International license.

a

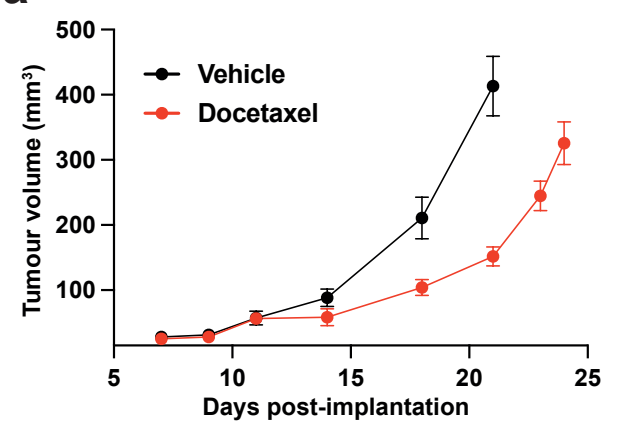

C

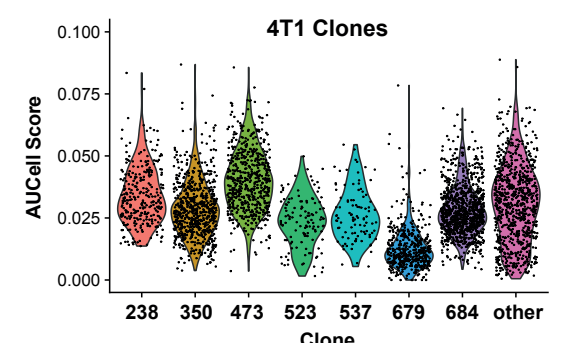

e

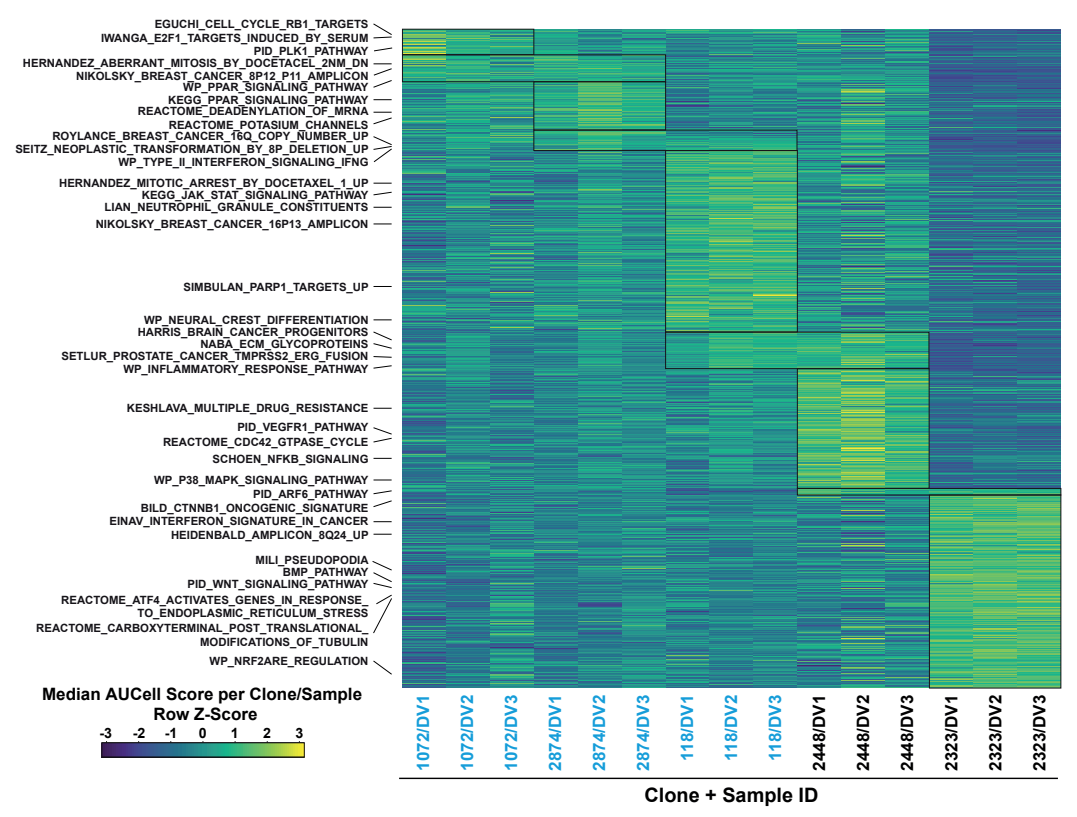

g

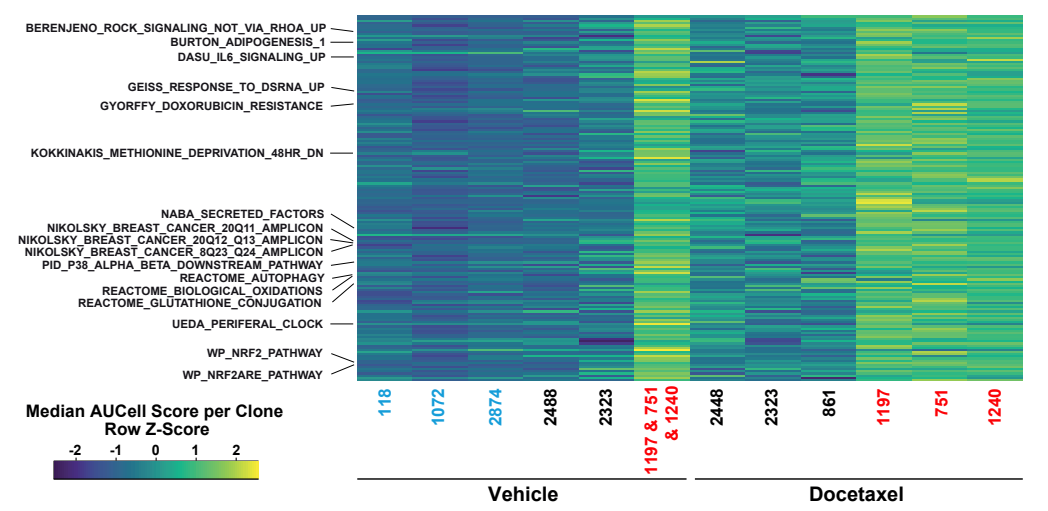

b All Cells Vehicle

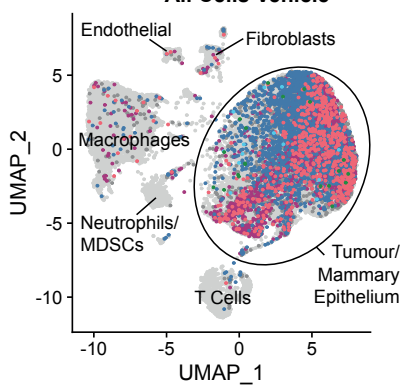

D2A1 Clones

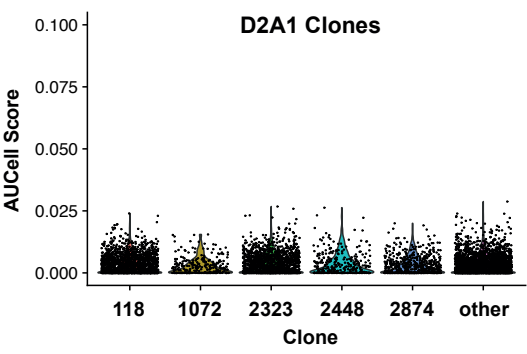

f
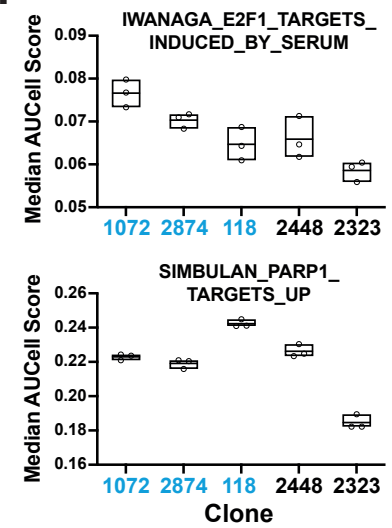

$\mathbf{h}$
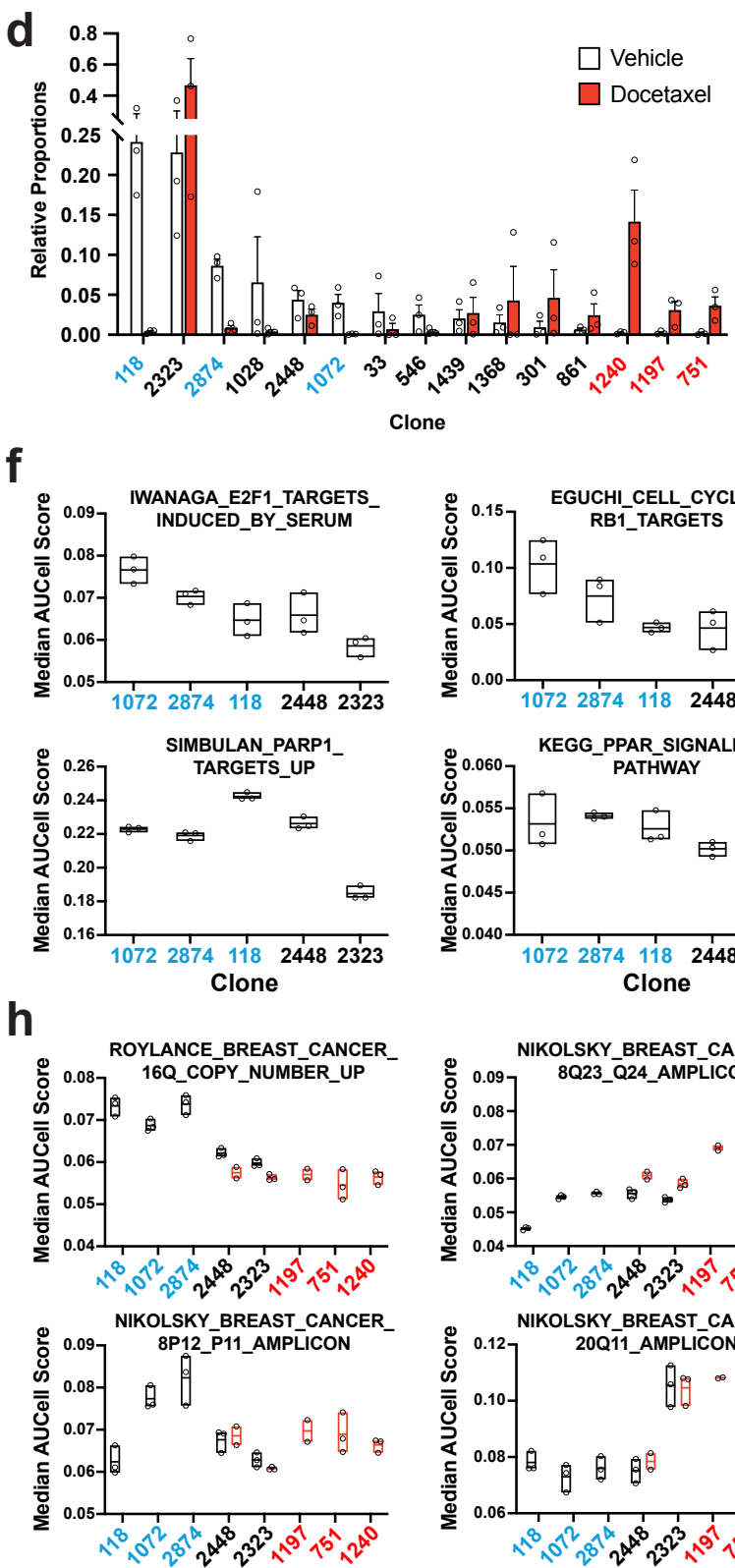

NIKOLSKY_BREAST_CANCER_

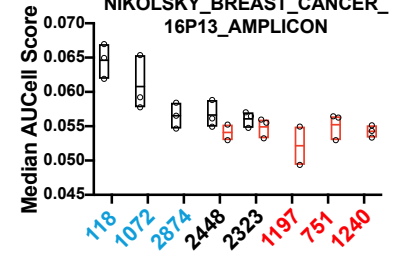

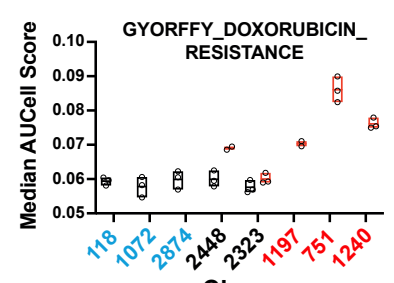

Clone
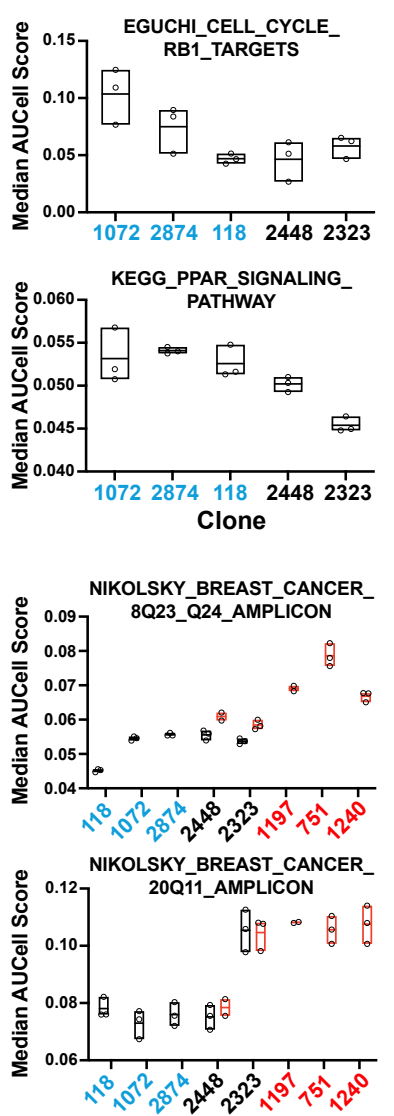

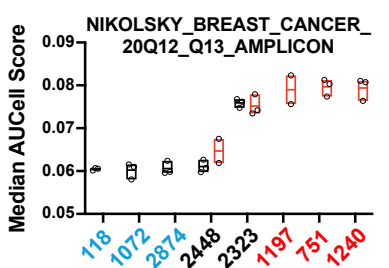

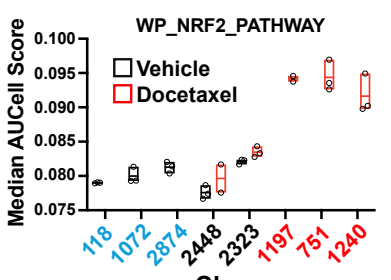

Clone 
bioRxiv preprint doi: https://doi.org/10.1101/2021.12.09.471927; this version posted December 10, 2021. The copyright holder for this preprint (which was not certified by peer review) is the author/funder, who has granted bioRxiv a license to display the preprint in perpetuity. It is made available under aCC-BY 4.0 International license.

Figure 4. Clonal transcriptomic signatures of response and resistance to taxane chemotherapy in the D2A1 mammary carcinoma model. a. D2A1 WILD-seq tumour growth curves with docetaxel treatment. D2A1 WILD-seq tumours were treated with docetaxel or vehicle from 7 days post-implantation for 2 weeks ( $n=5$ vehicle-treated mice, $n=4$ docetaxel-treated mice). Data represents mean \pm SEM. $\boldsymbol{b}$. scRNA-seq of docetaxel-treated D2A1 WILD-seq tumours. UMAP plots of vehicle-treated D2A1 WILD-seq D2A1 tumours and reclustered barcoded-tumour cells from vehicleand docetaxel-treated tumours. Combined cells from 3 mice per condition are shown. Cells for which a WILD-seq clonal barcode is identified are shown as dark grey or coloured spots. Cells which belong to five selected clonal lineages are highlighted. c. Comparison of EMT status of major 4T1 and D2A1 WILD-seq clones. Violin plot of AUCell scores from vehicle-treated tumour cells generated using the HOLLERN_EMT_BREAST_TUMOR_DN ${ }^{54}$ gene set, a set of genes that have low expression in murine mammary tumours of mesenchymal histology. 4T1 WILD-seq clones exhibit varying levels of expression of this geneset whereas D2A1 WILD-seq clones have consistently low levels of expression of these genes. d. Clonal representation. Proportion of tumour cells assigned to each clonal lineage based on the WILD-seq barcode ( $n=3$ tumours per condition). Clones which make up at least $2 \%$ of the assigned tumour cells under at least one condition are plotted. The most sensitive clones to docetaxel treatment 118, 2874 and 1072 are highlighted in blue and the most resistant clones 1240, 1197 and 751 are highlighted in red. Data represents mean \pm SEM. e. Clonal transcriptomic signatures from vehicle-treated tumours. Heatmap of median AUCell scores per sample for each of the five most abundant clones. All gene sets which showed consistent and statistically significant enrichment (combined fisher p-value $<0.01 \&$ mean $\log _{2}$ enrichment $>0.1$ ) in at least one of these clones are illustrated. f. Selected gene sets whose expression is associated with sensitivity to docetaxel. Median AUCell scores per sample for each of the five most abundant clones is plotted. $\mathbf{g}$. Transcriptomic signatures associated with resistance to docetaxel. For vehicle-treated tumours, resistant clonal lineages identified by barcodes 1197, 751 and 1240 were combined to have enough cells for analysis. Gene sets with significantly enriched expression in these resistant clones in vehicletreated tumours were determined (adjusted p-value $<0.01 \& \log _{2}$ enrichment $>0.1$ ). A heatmap of median AUCell scores per clone, per condition of these resistance-associated gene sets is plotted. $\mathbf{h}$. Selected gene sets whose expression is enriched or depleted in resistant clones. Median AUCell scores per clone, per sample are plotted for samples with at least 20 cells per clone. Due to changes in clonal abundance with treatment some clones can only be assessed under vehicle- or docetaxeltreated conditions. 
bioRxiv preprint doi: https://doi.org/10.1101/2021.12.09.471927; this version posted December 10, 2021. The copyright holder for this

preprint (which was not certified by peer review) is the author/funder, who has granted bioRxiv a license to display the preprint in perpetuity. It is made available under aCC-BY 4.0 International license.

Up in bulk analysis only

Down in bulk analysis only

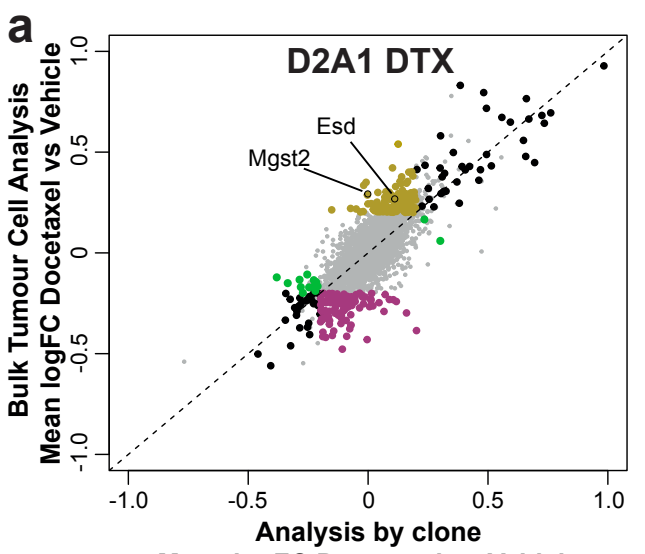

Mean logFC Docetaxel vs Vehicle
Change in clonal analysis only

Change in both analyses

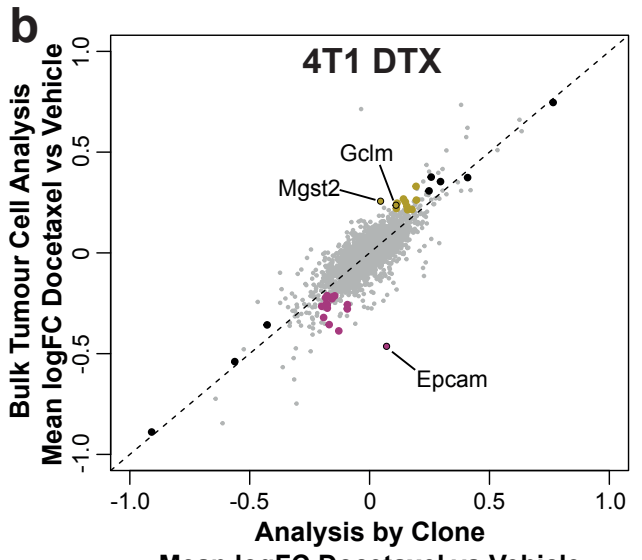

Mean logFC Docetaxel vs Vehicle

e

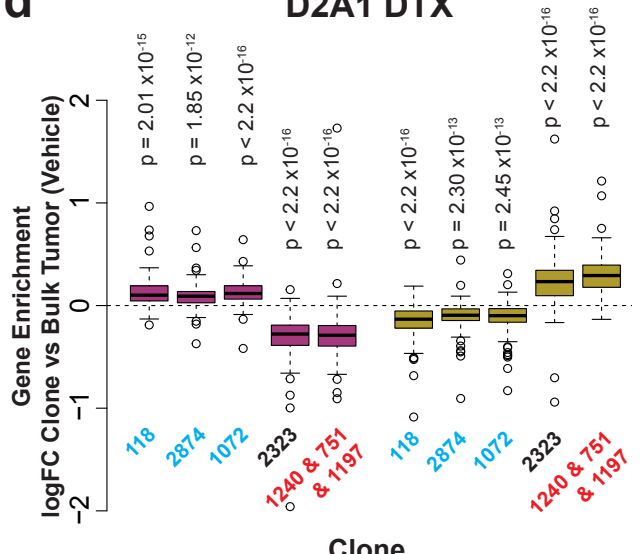

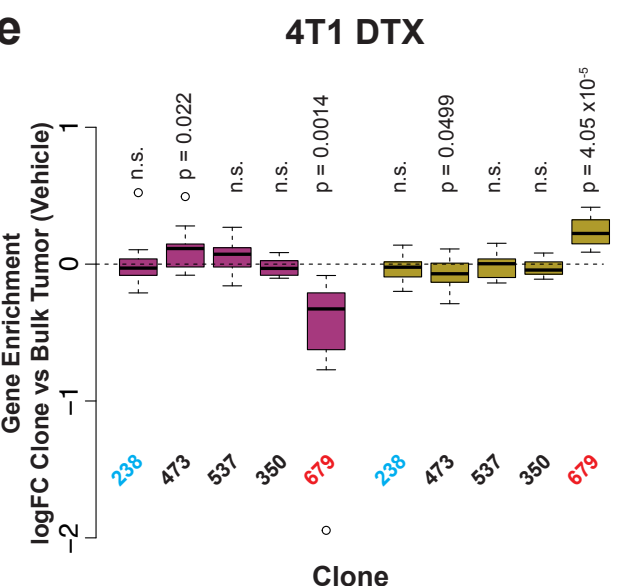

Clone

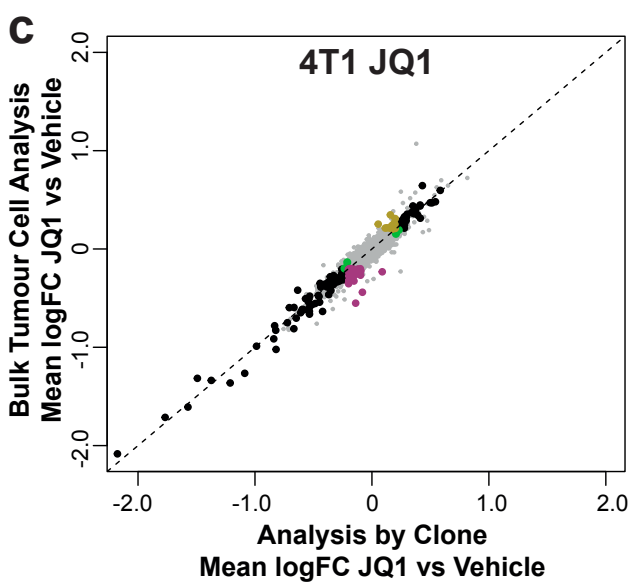

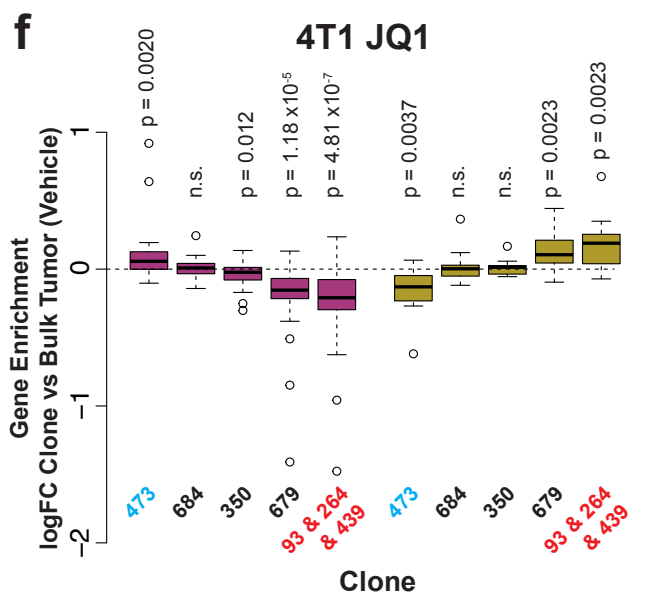

Figure 5. WILD-seq 
bioRxiv preprint doi: https://doi.org/10.1101/2021.12.09.471927; this version posted December 10, 2021. The copyright holder for this preprint (which was not certified by peer review) is the author/funder, who has granted bioRxiv a license to display the preprint in perpetuity. It is made available under aCC-BY 4.0 International license.

Figure 5. Delineating the contribution of clonal abundance to gene expression changes upon drug treatment. a. Comparison of differential gene expression analysis in bulk tumour cells and intra-clonal changes in gene expression. Differential gene expression was performed for all barcoded tumour cells irrespective of clonal lineage comparing chemotherapy-treated and vehicletreated cells (bulk tumour cell analysis). Alternatively differential gene expression was performed for each individual clone separately and the results combined to identify genes which robustly undergo intra-clonal changes in expression (analysis by clone). Whereas bulk tumour cell analysis will identify changes in overall gene expression due to both changes in clonal abundance and changes within the cells, analysis by clone enables us to delineate exclusively induced cellular changes in gene expression. Log $_{2}$ fold change in expression as determined by each of these analysis methods is plotted. Genes with significant changes in expression with chemotherapy ( $p$-value $<0.05$, logFC $<-0.2$ or $>0.2$ ) are highlighted based on the method under which they were identified. Genes identified as significantly changing by one method only met neither logFC nor $p$-value cutoffs in the alternative method. $\mathbf{b}$. Changes in gene expression that are identified by bulk tumour cell analysis only can attributed to changes in clonal abundance. The expression of genes which were identified as differential expressed after chemotherapy only in the bulk tumour cell analysis was assessed across clonal lineages at baseline. Baseline gene enrichment for each clone was determined as described previously by comparing cells of a specific clonal lineage to all barcoded tumour cells within the same vehicletreated sample or experiment. Gene enrichment values for all genes with differential expression only in the bulk tumour cell analysis were plotted. As expected, genes down-regulated in bulk analysis have lower expression in resistant clones, whereas genes up-regulated in bulk analysis are enriched in resistant clones. $p$-values represent a one sample t-test vs a theoretical mean of 0 . 
bioRxiv preprint doi: https://doi.org/10.1101/2021.12.09.471927; this version posted December 10, 2021. The copyright holder for this

a preprint (which was not certified by peer review) is the authorlander, who has granted bioRxiv a license to display the preprint in

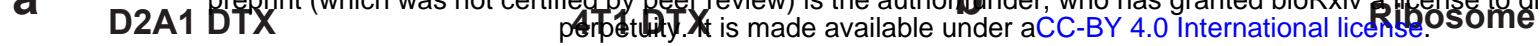
resistance genes resistance genes

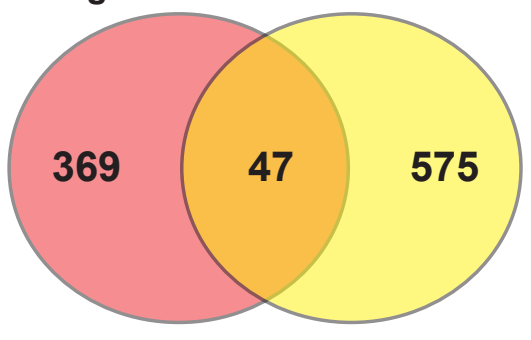

4T1/D2A1 DTX resistance genes d NRF2-target genes

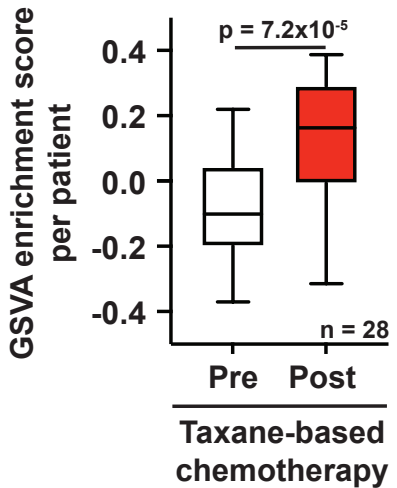

Glutathione conjugation

Glutathione-mediated detoxification

MYC CHEA (CHIP-targets)

Glutathione metabolism

Biological Oxidations

Oxidative stress induced gene expression via Nrf2 NFE2L2/NRF2 CHEA (CHIP-targets)

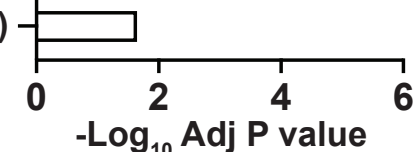

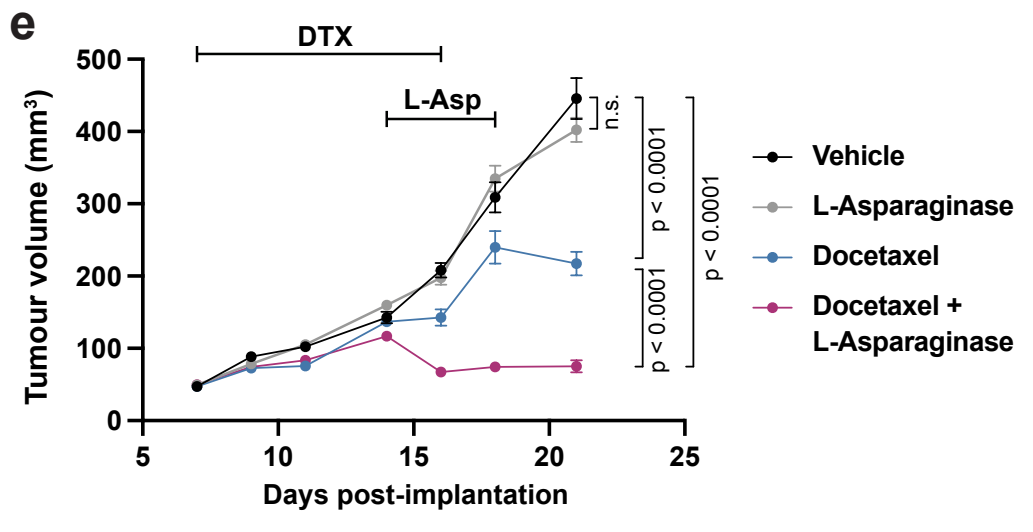

Figure 6. WILD-seq 
Figure 6. Convergent WILD-seq analysis across models identifies redox defense as a mediator of taxane resistance. a. Overlap of genes associated with resistance between the D2A1 and 4T1 WILD-seq models. 4T1 resistance genes were defined as those that were significantly enriched in resistant clone 679 but not in sensitive clone $238(p<0.05)$. D2A1 resistance genes were defined as those that were significantly enriched in combined resistant clones 1240, 751 and 1197 but not in sensitive clones 118, 2874 or $1072(p<0.05)$. In all cases in resistance genes were defined from vehicle treated tumours $b$. Gene set enrichment analysis of common resistance genes. Gene set enrichment was performed using Enrichr ${ }^{51-53}$ for the human orthologs of the 47 common resistance genes identified in a. Adjusted p-values for a subset of significant gene sets are plotted. $\mathbf{c}$. Expression of our identified resistance genes is increased in human breast tumours following docetaxel treatment. Expression of our 47 common resistance genes was assessed in human breast cancer samples taken before and after taxane-based neoadjuvant chemotherapy (GSE28844) ${ }^{38}$. GSVA enrichment scores for our gene set was calculated for samples from 28 patients for which matched preand post-treatment gene expression data were available. Patients received one of three taxanecontaining treatment regimens; Regimen A: Epirubicin $90 \mathrm{mg} / \mathrm{m}^{2}$-Cyclophosphamide $600 \mathrm{mg} / \mathrm{m}^{2}, 3$ cycles bi-weekly and Paclitaxel $150 \mathrm{mg} / \mathrm{m}^{2}$-Gemcitabine $2500 \mathrm{mg} / \mathrm{m}^{2}, 6$ cycles bi-weekly \pm weekly Herceptin $4 \mathrm{mg} / \mathrm{Kg}$ during the first week, $2 \mathrm{mg} / \mathrm{Kg}$ for the remaining 11 cycles. Regimen B: Doxorubicin $60 \mathrm{mg} / \mathrm{m}^{2}$-Pemetrexed $500 \mathrm{mg} / \mathrm{m}^{2}, 4$ cycles tri-weekly and Docetaxel $100 \mathrm{mg} / \mathrm{m}^{2}, 4$ cycles tri-weekly. Regimen C: Doxorubicin $60 \mathrm{mg} / \mathrm{m}^{2}$-Cyclophosphamide $600 \mathrm{mg} / \mathrm{m}^{2}, 4$ cycles tri-weekly and Docetaxel $100 \mathrm{mg} / \mathrm{m}^{2}$, 4 cycles tri-weekly. Expression of our common resistance gene set was significantly increased after chemotherapy in human samples. $p$-value calculated by paired t-test. d. NRF2-target genes are upregulated in human patients following neoadjuvant chemotherapy. GSVA enrichment scores for NRF2-target genes (NFE2L2 CHEA consensus CHIP-targets) were calculated for samples from 28 patients in the GSE28844 dataset for which pre- and post-treatment gene expression data were available. $p$-values calculated by paired t-test. e. Docetaxel-resistant tumours are collaterally sensitive to L-asparaginase. D2A1 WILD-seq tumours were treated with 3 doses of $12.5 \mathrm{mg} / \mathrm{kg}$ docetaxel (days 7,9,11 post-implantation) and 2 doses of $10 \mathrm{mg} / \mathrm{kg}$ docetaxel (days 14,16 post-implantation). From day 14 post-implantation mice were also treated daily with L-asparaginase. Statistical comparisons were performed between tumour volumes from different conditions at day 21 post-implantation. $p$-values were calculated by t-test, $n=10$ animals per condition. 